\title{
Three-dimensional crack initiation, propagation, branching and junction in non-linear materials by an extended meshfree method without asymptotic enrichment
}

\author{
Stéphane Bordas ${ }^{a}$ Timon Rabczuk ${ }^{b}$ Goangseup Zi ${ }^{c, *, 1}$ \\ ${ }^{a}$ Lecturer (assistant professor), Civil Engineering Department, University of \\ Glasgow, Rankine building, G12 8LT, Glasgow, UK; Tel: +44(0)141330 4075; \\ bordas@civil.gla.ac.uk \\ ${ }^{\mathrm{b}}$ Senior Lecturer, Department of Mechanical Engineering, University of \\ Canterbury, Christchurch, New Zealand; Tel: +64-3-364-8836; \\ Timon.Rabczuk@canterbury.ac.nz \\ ${ }^{\mathrm{c}}$ Assistant professor, Department of Civil \& Environmental Engineering, Korea \\ University, 5 Ga 1, An-Am Dong, Sung-Buk Gu, Seoul, 136-701, Korea; Tel.: \\ +82-2-3290-3324; g-zi@korea.ac.kr
}

\begin{abstract}
This paper presents a three-dimensional, extrinsically enriched meshfree method for initiation, branching, growth and coalescence of an arbitrary number of cracks in non-linear solids including large deformations, for statics and dynamics. The novelty of the methodology is that only an extrinsic discontinuous enrichment and no near-tip enrichment is required. Instead, a Lagrange multiplier field is added along the crack front to close the crack. This decreases the computational cost and removes difficulties involved with a branch enrichment. The results are compared to experimental data, and other simulations from the literature to show the robustness and accuracy of the method.
\end{abstract}

Key words: eXtended Element Free Galerkin Method (XEFG), discontinuous enrichment, cohesive cracks, Lagrange multipliers, dynamic fracture, large deformations, high velocity impact

\footnotetext{
* Corresponding author

Email address: g-zi@korea.ac.kr (Goangseup Zi).

1 The third author would like to thank the Agency for Defense Development (ADD) for grant ADD-06-05-06.
} 


\section{Introduction}

To model growing cracks in numerical methods such as finite element and meshfree methods, numerous techniques have been developed and those may be classified into two categories: the strong discontinuity approach and the weak discontinuity approach. In the former, cracks are modeled as discontinuities in the displacement field. In the latter, cracks are smeared within a certain region in which the deformation is localized. ${ }^{2}$ Recently, the strong discontinuity approach is of more interest than the weak discontinuity approach because one can study the behavior of solids near the sharp crack front with better resolution using the strong discontinuity approach.

Among the many methods of the strong discontinuity approach, the extended finite element method (XFEM) [1] is one of the most versatile and accurate. This method has been successfully applied to static problems in two and three dimensions, (see e.g $[2,3,4,5,6,7,8,9]$ ) and to dynamic problems [10, $11,12]$ in two dimensions and three dimensions including contact along the crack faces, and small-scale plasticity [13]. The extended finite element method is now utilized in industrial settings to assess damage tolerance of complex structures $[14,15,16]$ and open source $\mathrm{C}++$ libraries are available, such as $[17,18]$. Note that the stress field in the XFEM is not smooth because the method is an extended version of the standard finite element method as it is implied from its name.

Meshfree methods benefit from a higher order of continuity which naturally smoothes the stress field in the crack tip region. Fracture simulation work in two and three dimensions using meshfree methods is extensive [19, 20, $21,22,23]$, in which discontinuities are treated by the visibility criterion or some modifications of it. Other novel approaches which were able to treat kinked and curved cracks were proposed by Ventura et al. [24]. They enrich the moving least squares (MLS) basis functions around the crack tip and improve significantly the convergence behavior. ${ }^{3}$

Rabczuk et al. proposed the extended element free Galerkin (XEFG) method for cohesive crack initiation, growth and junction in two and three dimensional statics and dynamics, but the closure of the crack along the front is ensured through near-tip enrichment which vanishes along this front [25, 26, 27]. As noted in [26], the polar coordinate system along a crack front is not well-defined at the kinks. While the fields spanning the space of linear elastic fracture

\footnotetext{
2 in this approach, the so-called localization limiter must be introduced to keep the consistency of the energy release rate during the crack growth.

3 in these papers, the MLS basis is enriched extrinsically by adding special functions to the approximation. This is to be opposed to intrinsic enrichment at the level of the MLS basis
} 
mechanics solutions are known for small strains, this is the case neither in large strain nor for non-linear materials in general. This makes the selection of near-tip enrichment fields difficult in these circumstances, and calls for an alternate method. In this work, we do not use front enrichment, but only discontinuous enrichment; the crack is closed using a constraint field enforced by Lagrange multipliers. Similar accuracy compared to [26] is obtained on the examples treated. Furthermore, we add and test a crack branching algorithm.

The paper is arranged as follows: In the next section, we will describe the element-free Galerkin method. Section 3 describes the discontinuous enrichment employed and Section 4 the Lagrange multiplier method devised to close the crack fronts. The crack tracking algorithm follows, in Section 5 before the presentation of the governing equations in strong and weak form, in Section 6. The discretized version of the weak form is then presented in Section 7. We then show, in Section 8, several static and dynamic crack initiation/propagation problems and compare the results to experimental data or/and other numerical results in the literature, and close the work with some conclusions in Section 9.

\section{Element-free Galerkin (EFG) approximation}

The construction of the MLS approximation employed to describe the displacement field in the element-free Galerkin method is standard [19, 28, 29], and only recalled briefly for completeness. In the whole paper, we denote by $\Omega_{0}$ the body of interest in the reference configuration, and $\mathbf{X}=(X, Y, Z) \in \Omega_{0} \subset \mathbb{R}^{3}$ a point in the reference configuration. Let $\mathcal{W}$ be the set of all particles in $\Omega_{0}$. Particles (also called nodes, or points) are denoted by upper-case indices. Let $I$ be a particle, its approximation radius (smoothing length) is $h_{I}$, and its position in $\Omega_{0}$ is noted $\mathbf{X}_{I}$. For a point $\mathbf{X} \in \Omega_{0}$, the distance between $\mathbf{X}$ and particle $I$ is $r_{I}(\mathbf{X})=\left\|\mathbf{X}-\mathbf{X}_{I}\right\|$, where $\|\cdot\|$ is the Euclidian norm of $\mathbb{R}^{3}$. Each particle is associated with a weight (or kernel) function $W: \mathbb{R} \rightarrow \mathbb{R}$. In this paper, we used the cubic $B$-Spline. Continuity in meshfree methods is governed by the continuity of the kernel function $W$, in our case, the MLS shape functions derived below are $C_{2}$-continuous, as $W$. Defining the dimensionless distance $\bar{r}_{I}(\mathbf{X})=\frac{r_{I}(\mathbf{X})}{h_{I}}=\frac{\left\|\mathbf{X}-\mathbf{X}_{I}\right\|}{h_{I}}$, the weight function employed in this work 
writes

$$
\forall I \in \mathcal{W}, \forall \mathbf{X} \in \Omega_{0} \quad W\left(\bar{r}_{I}(\mathbf{X})\right)= \begin{cases}\frac{2}{3}-4 \bar{r}_{I}^{2}+4 \bar{r}_{I}^{3} & \text { for } \bar{r}_{I} \leq \frac{1}{2} \\ \frac{4}{3}-4 \bar{r}_{I}+4 \bar{r}_{I}^{2}-\frac{4}{3} \bar{r}_{I}^{3} & \text { for } \frac{1}{2}<\bar{r}_{I} \leq \frac{1}{2} \\ 0 & \text { for } \bar{r}_{I}>1\end{cases}
$$

The next required ingredient for the MLS approximation is the basis vector denoted by $\mathbf{p}=[1, X, Y, Z]$, which ensures that the MLS approximation can reproduce linear fields in $\mathbb{R}^{3}$. Denote by $\mathcal{I}_{I \mathbf{X}}$ the set of $N_{I \mathbf{X}}$ particles whose domain of influence contain a given point $\mathbf{X} \in \Omega_{0}$. The MLS shape function $\Phi_{I}$ associated with particle $I$ evaluated at point $\mathbf{X}$ is obtained by the matrix/vector product

$$
\begin{aligned}
\underbrace{\Phi_{I}(\mathbf{X})}_{N_{I} \times 1} & =\underbrace{\mathbf{p}^{T}}_{N_{I} \times 4}(\mathbf{X}) \cdot \underbrace{\mathbf{A}\left(\mathbf{X}, \mathbf{X}_{I}\right)^{-1}}_{4 \times 4} \cdot \underbrace{\mathbf{D}\left(\mathbf{X}, \mathbf{X}_{I}\right)}_{4 \times 1} \\
\mathbf{A}\left(\mathbf{X}, \mathbf{X}_{I}\right) & =\sum_{I \in \mathcal{I}_{I \mathbf{X}}} \mathbf{p}\left(\mathbf{X}_{I}\right) \mathbf{p}^{T}\left(\mathbf{X}_{I}\right) W\left(\bar{r}_{I}(\mathbf{X})\right) \\
\mathbf{D}\left(\mathbf{X}, \mathbf{X}_{I}\right) & =\sum_{I \in \mathcal{I}_{I \mathbf{X}}} \mathbf{p}\left(\mathbf{X}_{I}\right) W\left(\bar{r}_{I}(\mathbf{X})\right)
\end{aligned}
$$

With these definitions, the standard part $\left(C_{2}\right.$-continuous and linear-complete) of the MLS approximation (denoted by a superscript ${ }^{s}$ ) of the displacement field $\mathbf{u}^{s}$ at positive times $t$ can be written as

$$
\forall \mathbf{X} \in \Omega_{0}, \forall t>0, \quad \mathbf{u}^{s}(\mathbf{X}, t)=\sum_{I \in \mathcal{W}} \Phi_{I}(\mathbf{X}) \mathbf{u}_{I}^{s}(t)
$$

where the unknown coefficients $\mathbf{u}_{I}^{s}$ are functions of time $t$.

In addition to the fact that the order of continuity can be increased quite easily, meshfree methods have advantages over finite elements because of their smoothness and nonlocal interpolation character. Better stress distributions around the crack tip are obtained, leading to a non-oscillatory crack path.

\section{Discontinuous enrichment of the displacement field}

To model the discontinuity due to cracks, the displacement approximation is written as the sum of a standard $\left(\mathbf{u}^{s}\right)$ part as in Eq. (5) and an enriched part 
$\left(\mathbf{u}^{e}\right)$, discontinuous through the faces of the cracks

$$
\forall \mathbf{X} \in \Omega_{0}, \forall t>0, \quad \mathbf{u}(\mathbf{X}, t)=\mathbf{u}^{s}(\mathbf{X}, t)+\mathbf{u}^{e}(\mathbf{X}, t)
$$

Define $\mathcal{E}$ as the set of all the cracks in the domain and $\mathbf{u}_{\alpha}^{e}$ the enriched part (discontinuous part) of the displacement approximation due to crack $\alpha$. The enriched part of the displacement approximation due to all cracks in $\mathcal{E}$ is the sum of the discontinuous contribution associated with each crack:

$$
\forall \mathbf{X} \in \Omega_{0}, \forall t>0, \quad \mathbf{u}^{e}(\mathbf{X}, t)=\sum_{\alpha \in \mathcal{E}} \mathbf{u}_{\alpha}^{e}(\mathbf{X}, t)
$$

Let us now derive an expression for $\mathbf{u}_{\alpha}^{e}$. Let $\mathcal{W}_{\alpha}$ be the set of particles whose domain of influence is cut by crack $\alpha$, and $\Psi_{I}^{\alpha}$ be the enrichment function associated with particle $I$ and crack $\alpha$, discontinuous through this crack and defined in detail below. Define by $\boldsymbol{a}_{I}^{\alpha}$ the additional degrees of freedom for the enrichment $\Psi_{I}^{\alpha}$. The discontinuous part of the displacement approximation due to crack $\alpha \in \mathcal{E}$ writes

$$
\forall \mathbf{X} \in \Omega_{0}, \forall t>0, \quad \mathbf{u}_{\alpha}^{e}(\mathbf{X}, t)=\sum_{I \in \mathcal{W}_{\alpha}} \Phi_{I}(\mathbf{X}) \Psi_{I}^{\alpha}(\mathbf{X}) \boldsymbol{a}_{I}^{\alpha}(t)
$$

Note that the shape functions $\Phi_{I}$ in Eq. (8) need not be the same as $\Phi_{I}$ in Eq. (5) $[30,31,32]$, it will however be the case in the numerical examples to follow.

We now define the enrichment functions $\Psi_{I}^{\alpha}$. If a domain of influence is cut by a crack, it is enriched with the sign function given by

$$
\operatorname{sign}(x)=\left\{\begin{array}{c}
1 \text { for } x>0 \\
-1 \text { for } x<0
\end{array}\right.
$$

Let $\mathbf{n}$ be the crack normal and $\Gamma_{c}^{\alpha}$ represent the surface of crack $\alpha$. The choice of the orientation of the crack normal is completely arbitrary as long as it is consistent throughout the entire computation.

Let $\mathbf{X}_{\alpha}$ be a point on the surface $\Gamma_{c}^{\alpha}$ of crack $\alpha \in \mathcal{E}$ and $\mathbf{X}$ a point in $\Omega_{0}$. The sign of quantity $\mathbf{n} \cdot\left(\mathbf{X}-\mathbf{X}_{\alpha}\right)$ defines on which side of $\Gamma_{c}^{\alpha} \mathbf{X}$ is located. The distance from $\mathbf{X}$ to $\Gamma_{c}^{\alpha}$ is $\min _{\mathbf{X}^{\Gamma} \in \Gamma_{c}^{\alpha}}\left\|\mathbf{X}-\mathbf{X}^{\Gamma}\right\|$. The signed distance $f^{\alpha}(\mathbf{X})$ from $\mathbf{X}$ to crack $\alpha$ then writes

$$
\forall \mathbf{X} \in \Omega_{0}, \forall \mathbf{X}_{\alpha} \in \Gamma_{c}^{\alpha}, \quad f^{\alpha}(\mathbf{X})=\operatorname{sign}\left[\mathbf{n} \cdot\left(\mathbf{X}-\mathbf{X}_{\alpha}\right)\right] \min _{\mathbf{X}^{\Gamma} \in \Gamma_{c}^{\alpha}}\left\|\mathbf{X}-\mathbf{X}^{\Gamma}\right\|
$$




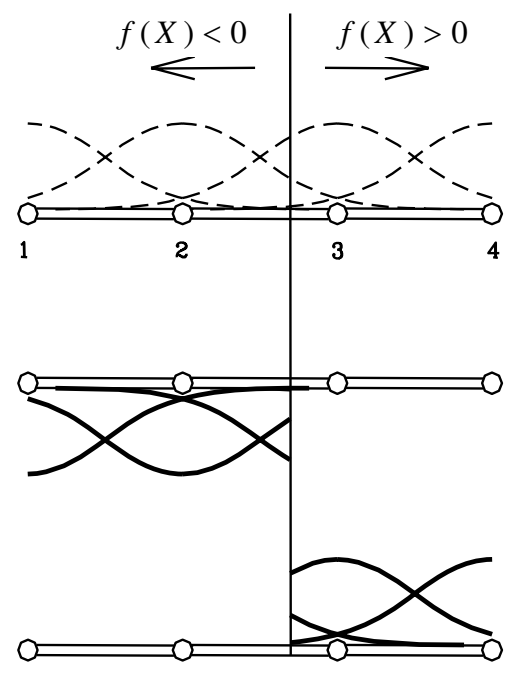

(a)

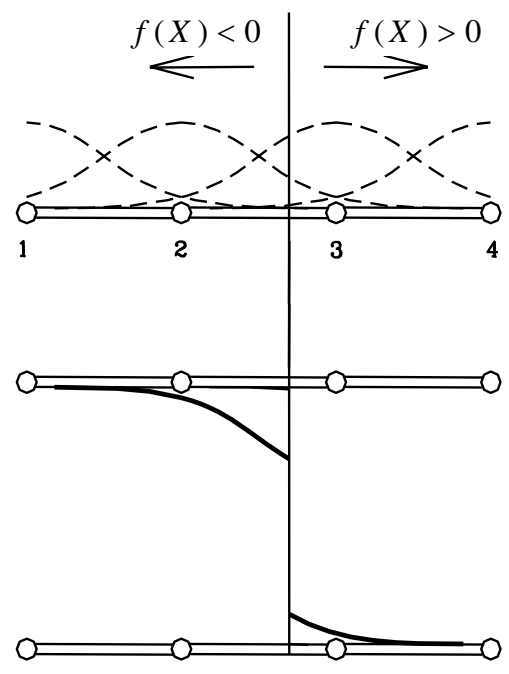

(b)

Fig. 1. The enriched displacement field in one dimension enriched (a) by just the sign of the signed distance function $f$ and (b) by the shifted sign of the signed distance function as in Eq. (11).

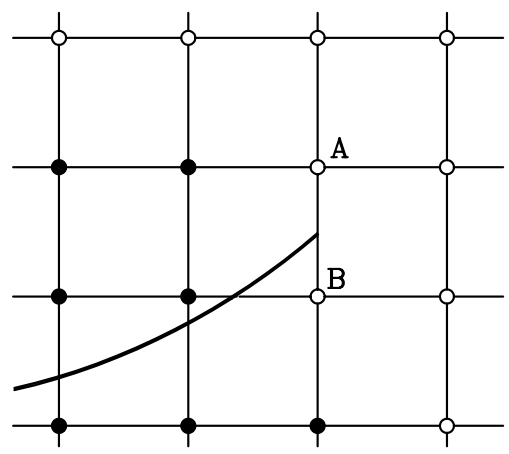

(a) finite element method

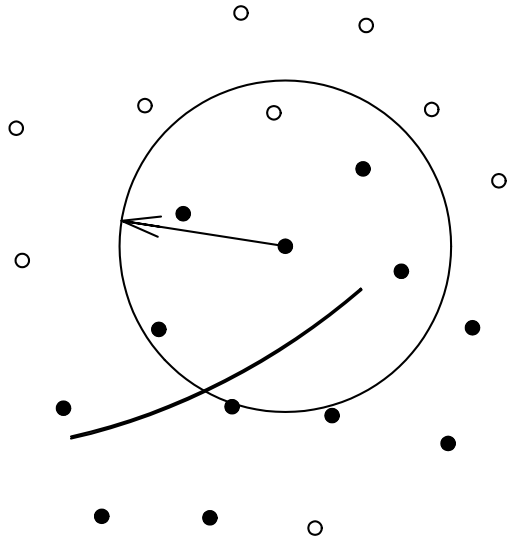

(b) meshless method

Fig. 2. The enrichment for the crack tip by using the step function in (a) the finite element method and (b) meshless methods; solids are enriched nodes and circles unenriched nodes.

The enrichment for crack $\alpha$ and particle $I$ writes

$$
\forall \alpha \in \mathcal{E}, \forall I \in \mathcal{W}, \forall \mathbf{X} \in \Omega_{0}, \quad \Psi_{I}^{\alpha}(\mathbf{X})=\operatorname{sign}\left[f^{\alpha}(\mathbf{X})\right]-\operatorname{sign}\left[f^{\alpha}\left(\mathbf{X}_{I}\right)\right]
$$

The enriched displacement field is shown in Fig. 1 for one dimensional case. Note that the enrichment function was shifted by its nodal values in Eq. (11). The use of the shifted enrichment function makes the enriched region narrower. The magnitude of the displacement jump is scaled by $\boldsymbol{a}_{I}^{\alpha}(t)$ in Eq. (8). 


\section{Enforcing crack closure along their front}

Let us now see how the cracks may be closed along their fronts without recourse to near-front enrichment in meshfree methods. When cracks are not discretized, i.e. when nodes are not present along the crack faces, as is the case in enriched FEM and meshfree methods, a strategy must be fashioned to close each crack along its front. Crack closure along the front is a natural outcome of near-front enrichment vanishing along the front, as in [26] for instance. In the context of the extended finite element method, a methodology was devised to close the crack without near-front enrichment [4, 33] as long as the crack tip is located on element edges. In $C_{0}$-FEM-based methods, the shape function associated with each node is only coupled with those of the nodes contained in its support. Imagine a crack tip in a two-dimensional finite element mesh; see Figure 2a, where the crack tip is positioned on the edge connecting nodes $A$ and $B$. Because the crack must close at its tip, i.e. the crack opening displacement must be zero, nodes $A$ and $B$ should not be enriched ${ }^{4}$. Figure $2 \mathrm{~b}$ shows the case of meshless methods. The domain of influence for a particle in meshless methods overlaps heavily with that of other particles. The idea presented in $[4,33]$ would be applicable if and only if the crack tips were located on the boundary of domains of influence, but never inside, i.e. if all domains of influence were either not cut by the crack, or completely cut by the crack, but never partially cut.

Recently, Zi et al. [34] developed a simple method to close the crack tip without near-tip enrichment. The method is similar to the technique developed for XFEM by Zi and Belytscko [35]; the domains of influence containing a crack tip are suitably adjusted so that the crack tip is placed on their edge. However, their approach is limited for two dimensional problems only and, as shown in Figure 3, supports must be artificially modified so as to be entirely split by the crack, and that the crack tip always lie on a support boundary, but never in its interior. Extending this to three dimensions is not straightforward. The crack front is composed of a series of segments that would need to be made conform with the boundaries of the spherical supports.

We propose the construction of a Lagrange multiplier field to close the cracks along their fronts. If only the sign function enrichment of Eq. (11) is used, the discontinuity $\Gamma_{\mathrm{c}}$ extends beyond the crack front, as depicted in Figure 4. To correctly model the crack, the discontinuity on the front $\Gamma_{\mathrm{c}}$ should vanish. Because the condition should be satisfied along a line in 3D space, the Lagrange multiplier field must be discretized. To avoid introducing additional nodes for

\footnotetext{
$\overline{4}$ if they were enriched with a function discontinuous through the crack interior, the displacement approximation would be discontinuous along line segment $[\mathrm{AB}]$, and the the opening of the crack would be non-zero.
} 


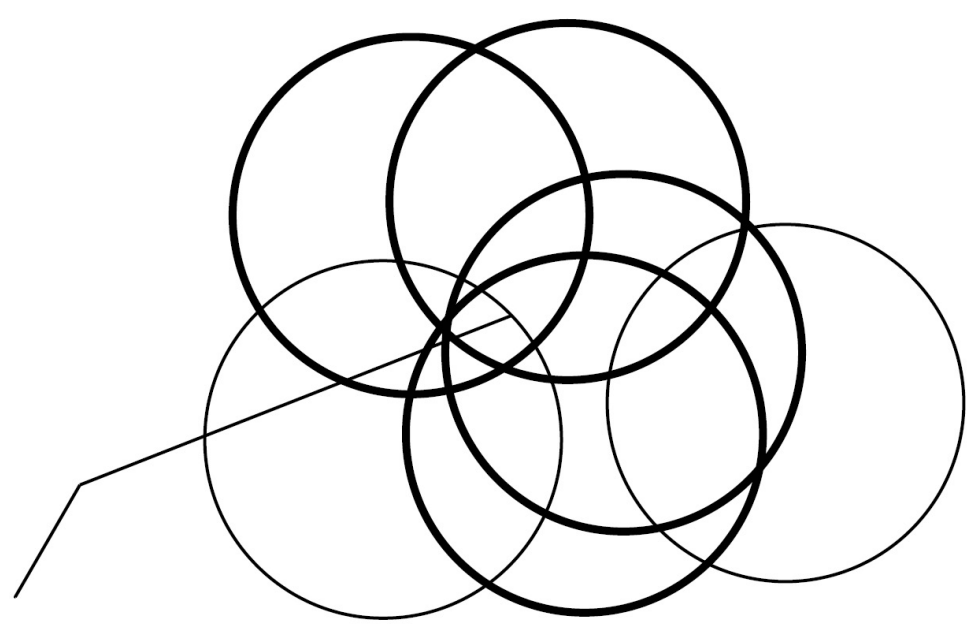

(a) Standard domains of influence: the heavy lines indicate supports which are partially cut by the crack, and would therefore lead to the artificial extension of the crack represented in Figure 4.

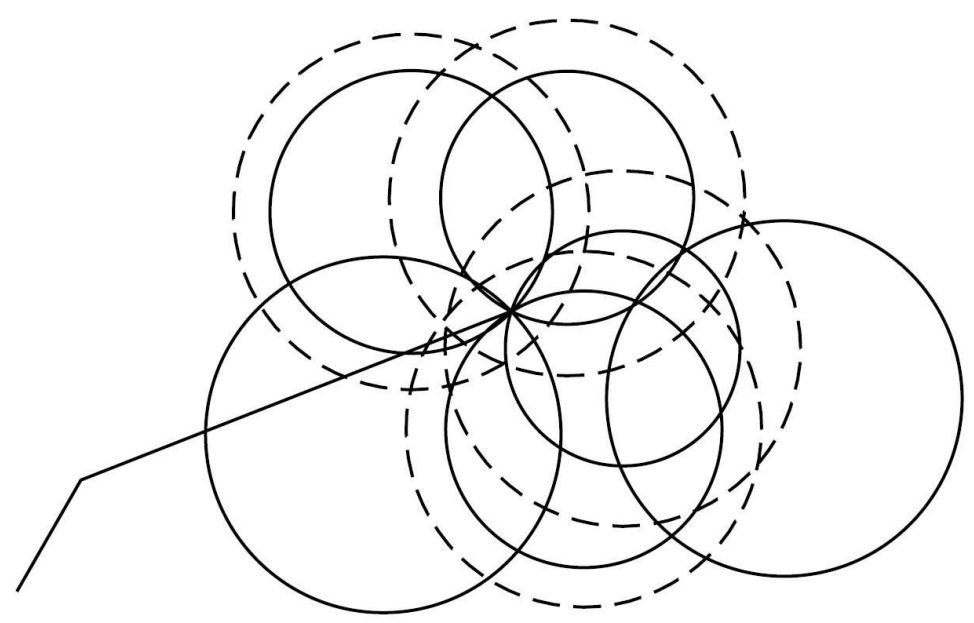

(b) Domains of influence are modified so that the tip is always on their boundary and that there exists no support that is partially cut by the crack.

Fig. 3. Method proposed by Zi et al. [34] to keep the crack tip on the boundaries of domains of influence. This methodology is difficult to scale up to three dimensions where the front is a broken line and the supports are spheres. 


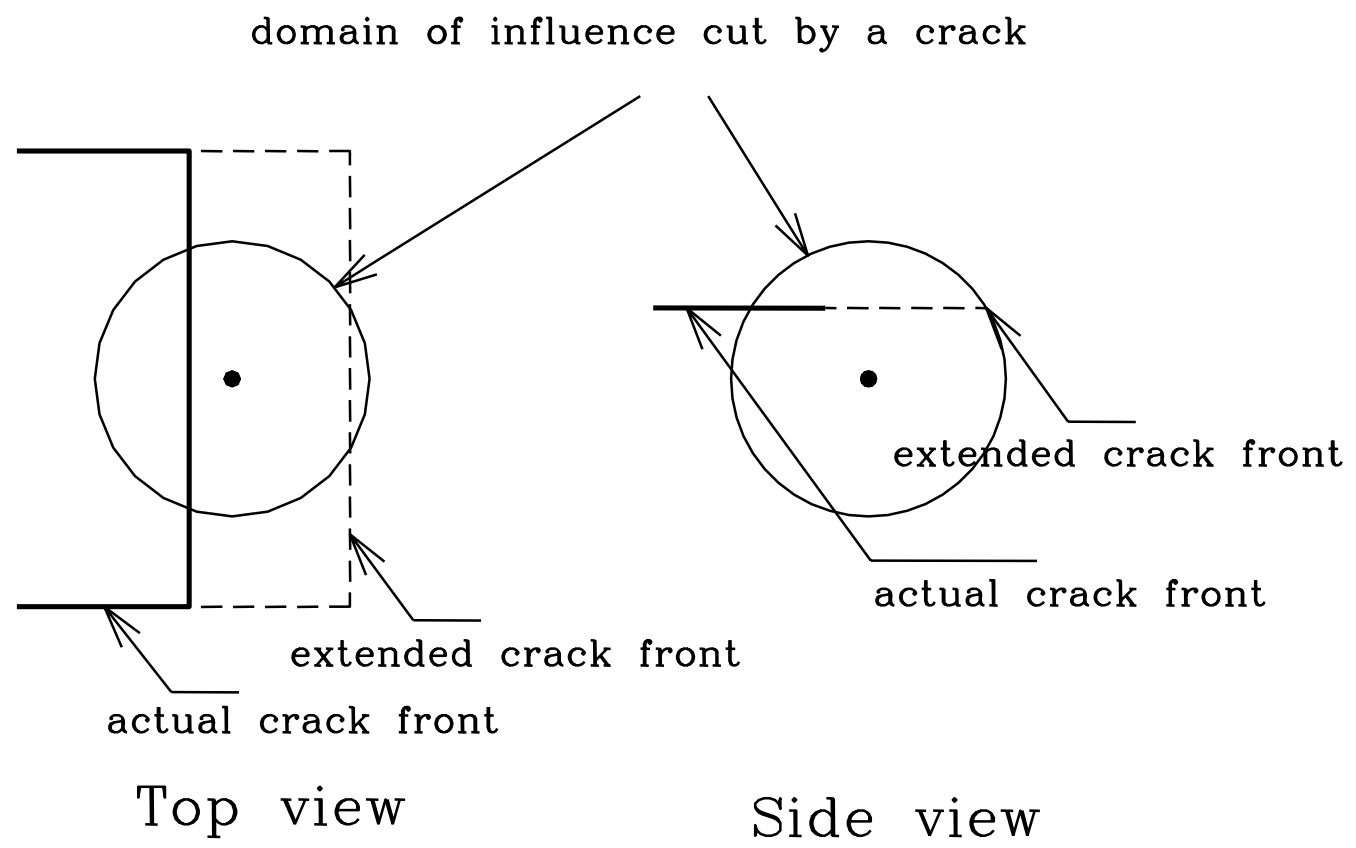

Fig. 4. When a domain of influence is cut by a crack, the particle is enriched with the Heaviside (jump) function. If no special care is taken, the crack artificially extends throughout the domain of influence of the enriched particle, which leads to an inaccurate crack representation. To avoid this, we add a Lagrange multiplier field along the crack front, to close it. 


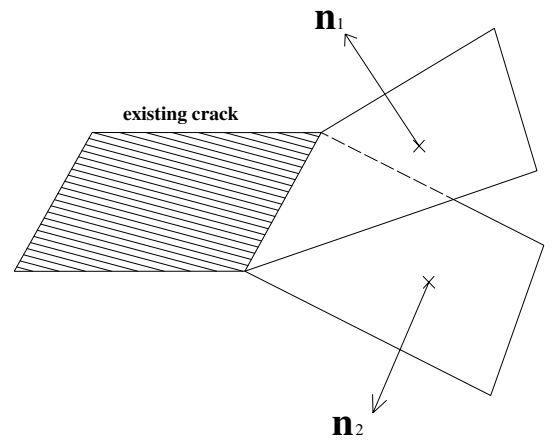

Fig. 5. Crack branching in a given background cell. If more than one Gauß point is found to loose stability ahead of an existing crack front, and the normals obtained by the eigenvalue analysis are "significantly" different, the crack branches. Cross symbols represent failed Gaußpoints ahead of the crack front.

the discretization of the Lagrange multipliers, we use the same shape functions as those for the domain partially cut by the crack. The detailed formulation is given later.

\section{Description of Cracks}

\subsection{Geometric description}

The cracks are described similarly as in [26]. The crack surfaces are nonplanar and represented by the union of planar segments obtained by slicing the tetrahedral background mesh by the failure planes obtained through material stability analysis (described in Section 5.2). These crack segments are either triangles or quadrangles depending on how the tetrahedron is split.

\subsection{Initiation and propagation of cracks}

We employ the loss of hyperbolicity (in dynamics) and loss of ellipticity (in quasi-statics) criterion for crack initiation and propagation, as proposed by Belytscko et al. [10]. Therefore, a crack is initiated or propagated if the minimum eigenvalue of the acoustic tensor $\boldsymbol{Q}$ is smaller or equal to zero:

$$
\min \operatorname{eig}(\boldsymbol{Q}) \leq 0 \text { with } \quad \boldsymbol{Q}=\mathbf{n} \cdot \mathbf{A} \cdot \mathbf{n}
$$

where $\mathbf{n}$ is the normal to the crack surface, $\mathbf{A}=\boldsymbol{C}^{t}+\boldsymbol{\sigma} \otimes \boldsymbol{I}, \boldsymbol{\sigma}$ is the stress tensor and $\boldsymbol{C}^{t}$ is the fourth order tangential modulus.

Note that we allow crack branching in a given background cell, as in Figure 
5. The loss of ellipticity/hyperbolicity is checked at the Gauß points ahead of the crack fronts; if two significantly different normal directions $\left(_{1}\right.$ and $_{2}$ in Figure 5) are found, the crack is assumed to branch.

\subsection{Measure of the crack opening and sliding}

Since the standard part, $\mathbf{u}^{s}$ of the displacement field is continuous everywhere, the jump in the displacement field is governed only by the enriched part $\mathbf{u}^{e}$ and is given by

$$
\llbracket \mathbf{u}(\mathbf{X}) \rrbracket=2 \sum_{\alpha \in \mathcal{E}} \sum_{I \in W_{\alpha}(\mathbf{X})} \Phi_{I}^{\alpha}(\mathbf{X}) \boldsymbol{a}_{I}^{\alpha}
$$

The normal part $\delta_{n}$, i.e. the crack opening and the tangential part $\llbracket \mathbf{u}(\mathbf{X}) \rrbracket_{\tau}$, the crack sliding is given by

$$
\begin{aligned}
\delta_{n} & =\mathbf{n} \cdot \llbracket \mathbf{u}(\mathbf{X}) \rrbracket \\
\delta_{t} & =\left\|\llbracket \mathbf{u}(\mathbf{X}) \rrbracket-\mathbf{n} \delta_{n}\right\|
\end{aligned}
$$

\subsection{Tracking the crack path}

The algorithmic procedure to track the crack path is detailed in Rabczuk et al. [26]. We extend the algorithm such that it can handle crack branching within the same background cell. Moreover, we allow multiple cracks to overlap during initiation (at the same time step ${ }^{5}$ ). Especially for problems with excessive cracking, this facilitates the implementation and also decreases computational cost.

Let us now consider branching and joining cracks as shown in Figures 6. The discontinuities are located using the signed-distance function that is called $f$ in the following. Let $\mathcal{W}_{b}^{1}$ be the set of nodes whose domain of influence is cut by the discontinuity $f^{1}(\mathbf{X})=0$ and $\mathcal{W}_{b}^{2}$ the corresponding set for $f^{2}(\boldsymbol{X})=0$. $\mathcal{W}_{b}^{3}=\mathcal{W}_{b}^{1} \cap \mathcal{W}_{b}^{2}$. By using the signed distance functions of the pre-existing and approaching crack, the signed distance function of the approaching crack is modified. Consider Figure 6. Three different subdomains have to be considered [5]: $\left(f^{1}<0, f^{2}<0\right),\left(f^{1}>0, f^{2}>0\right),\left(f^{1}>0, f^{2}<0\right)$ as in Figure 6b or $\left(f^{1}>0, f^{2}<0\right),\left(f^{1}>0, f^{2}>0\right),\left(f^{1}<0, f^{2}<0\right)$ as in Figure 6d. The

\footnotetext{
$\overline{5}$ in contrast to our method in [26] where we did not allow overlapping of simultaneously initiated cracks, see Figure 7.
} 


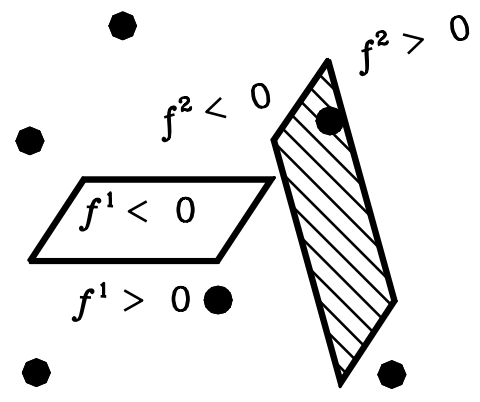

(a)

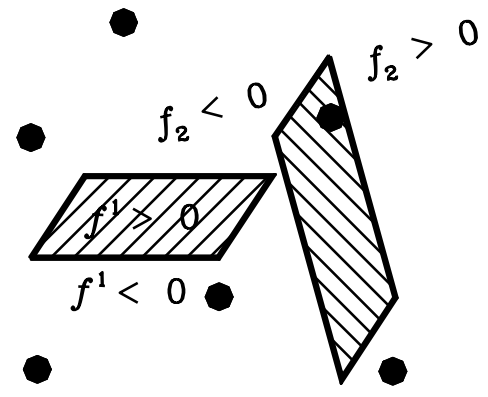

(c)

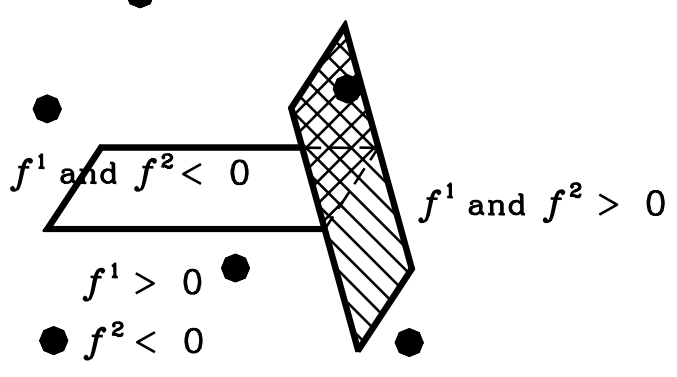

(b)

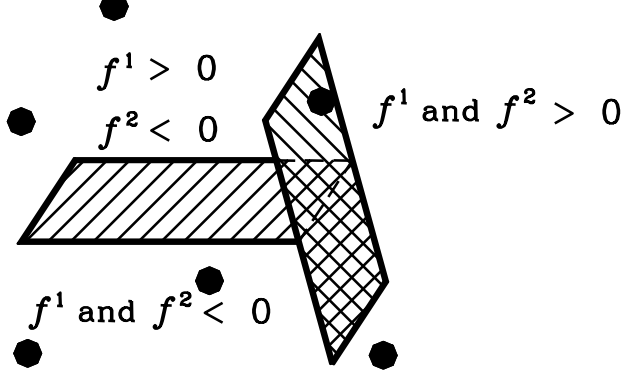

(d)

Fig. 6. The change of the signed distance function when one crack joins with another, in which the signed distance function of cracks in (a) and(b) is changed to that in (c) and (d), respectively; the hatched plane represents the side with positive sign.
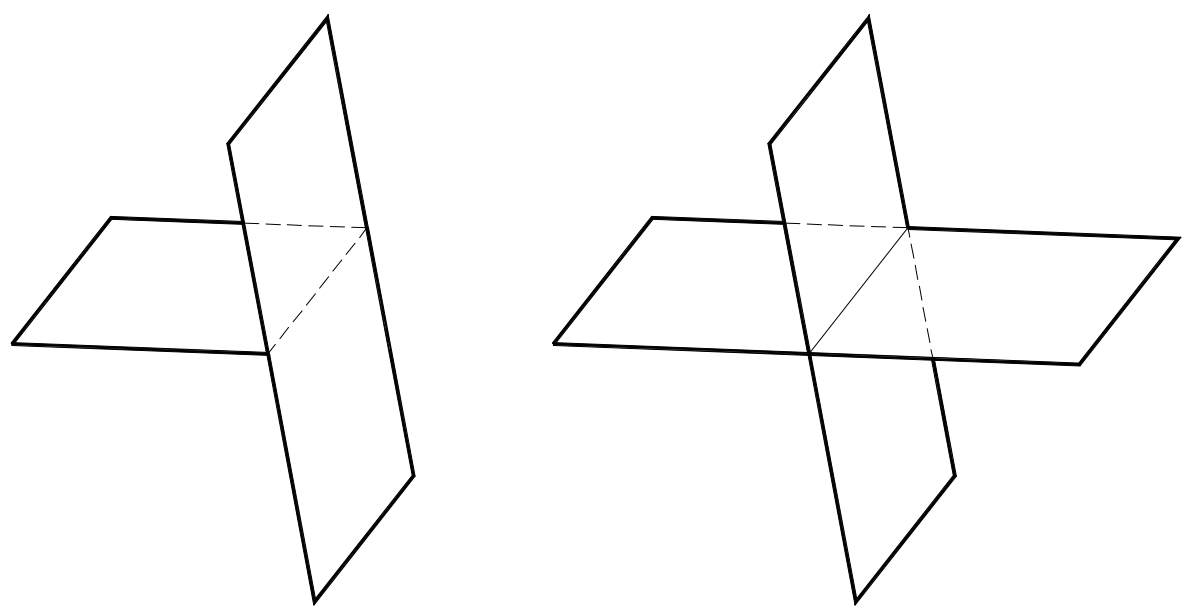

Fig. 7. Allowing overlap during "simultaneous" crack initiation. Left: two simultaneously initiating cracks are cut at their intersection, as in [26]. Right: the two cracks are allowed to overlap, this is the formulation adopted in this paper. 
signed distance function of crack 1 of a point $\mathbf{X}$ is then obtained by:

$$
f^{1}(\mathbf{X})=\left\{\begin{array}{l}
f_{0}^{1}(\mathbf{X}), \text { if } f_{0}^{2}\left(\mathbf{X}^{(1)}\right) f_{0}^{2}(\mathbf{X})>0 \\
f_{0}^{2}(\mathbf{X}), \text { if } f_{0}^{2}\left(\mathbf{X}^{(1)}\right) f_{0}^{2}(\mathbf{X})<0
\end{array}\right.
$$

in which $f_{0}^{1}(\mathbf{X}), f_{0}^{2}(\mathbf{X})$ represent the signed distance functions of cracks 1 and 2 without consideration of the junction, respectively, and $\mathbf{X}^{(1)}$ is any point on crack 1 . The approximation for a branching crack reads then

$$
\mathbf{u}(\mathbf{X})=\sum_{I \in \mathcal{W}(\mathbf{X})} \Phi_{I}(\mathbf{X}) \mathbf{u}_{I}+\sum_{\alpha=1}^{n_{c}} \sum_{I \in \mathcal{W}_{b}(\mathbf{X})} \Phi_{I}(\mathbf{X}) H\left(f_{I}^{(\alpha)}(\mathbf{X})\right) \mathbf{a}_{I}^{(\alpha)}
$$

where $n_{c}$ is the number of cracks

\section{Governing equations}

\subsection{The momentum equation and the boundary conditions}

Defining $\Gamma_{0}^{c}$ as the union of all the crack surfaces, and denoting $\nabla_{0}$ the gradient operator in the reference configuration,the strong form of the momentum equation in the total Lagrangian description is given by

$$
\varrho_{0} \ddot{\mathbf{u}}=\nabla_{0} \cdot \mathbf{P}+\varrho_{0} \boldsymbol{b} \quad \text { in } \quad \Omega_{0} \backslash \Gamma_{0}^{c}
$$

with boundary conditions:

$$
\begin{aligned}
\mathbf{u}(\mathbf{X}, t) & =\overline{\mathbf{u}}(\mathbf{X}, t) \quad \text { on } \quad \Gamma_{0}^{u} \\
\mathbf{n}_{0} \cdot \mathbf{P}(\mathbf{X}, t) & =\overline{\boldsymbol{t}}(\mathbf{X}, t) \quad \text { on } \quad \Gamma_{0}^{t} \\
\mathbf{n}_{0} \cdot \mathbf{P}^{-} & =\mathbf{n}_{0} \cdot \mathbf{P}^{+}=\boldsymbol{t}_{c 0} \quad \text { on } \Gamma_{0}^{c} \\
\boldsymbol{t}_{c 0} & =\boldsymbol{t}_{c 0}(\llbracket \mathbf{u} \rrbracket) \text { on } \quad \Gamma_{0}^{c}
\end{aligned}
$$

where $\varrho_{0}$ is the initial mass density, $\ddot{\mathbf{u}}$ is the acceleration, $\mathbf{P}$ denotes the nominal stress tensor, $\boldsymbol{b}$ designates the body force, $\overline{\mathbf{u}}$ and $\overline{\boldsymbol{t}}$ are the prescribed displacement and traction, respectively, $\mathbf{n}_{0}$ is the outward normal to the domain and $\Gamma_{0}^{u} \cup \Gamma_{0}^{t} \cup \Gamma_{0}^{c}=\Gamma_{0},\left(\Gamma_{0}^{u} \cap \Gamma_{0}^{t}\right) \cup\left(\Gamma_{0}^{t} \cap \Gamma_{0}^{c}\right) \cup\left(\Gamma_{0}^{c} \cap \Gamma_{0}^{u}\right)=\emptyset$. Moreover, we assume that the stresses $\mathbf{P}$ are bounded on the crack surface $\Gamma_{0}^{c}$. Since the stresses are not well defined along the crack, the crack surface $\Gamma_{0}^{c}$ is excluded from the domain $\Omega_{0}$ which is considered as an open set. 


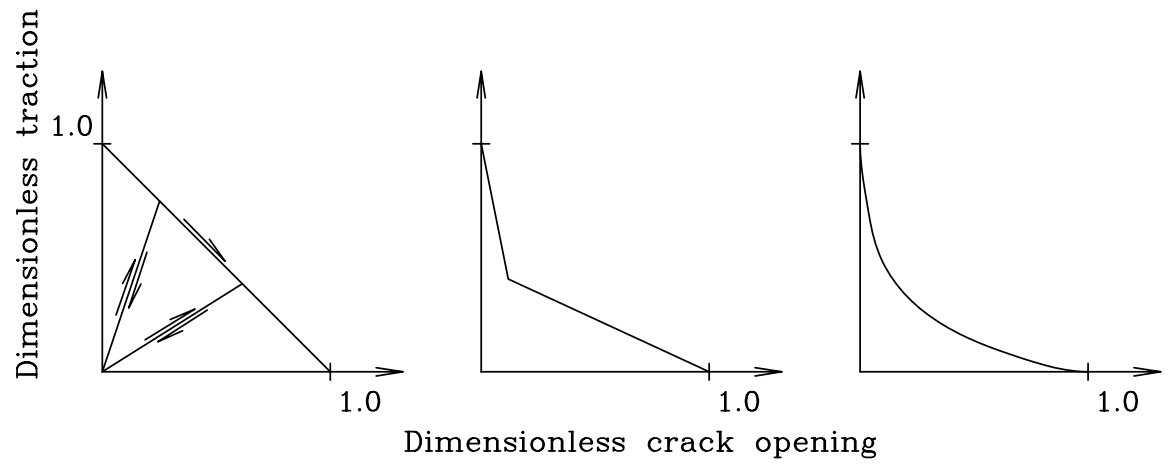

Fig. 8. The types of the cohesive laws frequently used in practice; (a) linear (or triangular), (b) bilinear and (c) exponential cohesive laws. 


\subsection{Cohesive cracks}

We exclusively used initially rigid cohesive models as shown in Figure 8 for one-dimensional stress-displacement laws. When a potential for the cohesive crack is defined, the unidirectional stress-displacement relation can be extended to general mixed mode problems, as in $[36,37,38]$. Note that if only one-dimensional cohesive models for mode-I fracture are employed, traction continuity is in general violated. We only employed cohesive models that fulfill the traction continuity condition, i.e. the cohesive traction $\mathbf{t}_{c}=\mathbf{n} \cdot \boldsymbol{\sigma}$ at crack initiation is compatible with the stress state at crack initiation. We note that the fulfillment of traction continuity is a complementary problem and cannot simply be enforced by Lagrange multipliers since the traction cannot exceed the tensile strength of the material.

Difficulties would occur at crack initiation due to the infinite slope of the tangent, especially when unloading occurs at an early stage. However, because Gauß points for the integration of the traction are never placed at the crack front, it is not a problem in practice.

\section{Discretized Equations}

\subsection{Discretization}

The weak form of the momentum equation is given by

$$
\delta W=\delta W_{\text {int }}+\delta W_{\text {kin }}-\delta W_{\text {ext }}-\delta W_{\text {coh }}+\delta W_{\mathrm{L}}
$$

in which $\delta W_{\text {int }}, \delta W_{\text {kin }}, \delta W_{\text {ext }}, \delta W_{\text {coh }}$ are the parts composing the virtual work of the internal stress, the inertia force, the external traction and the cohesive traction, respectively; $\delta W_{\mathrm{L}}$ is introduced to close the crack at its crack front. The four parts of the virtual work write ${ }^{6}$

$\overline{6 \text { recall }}$ that $\Gamma_{0}^{c}$ is the union of all crack surfaces. 


$$
\begin{aligned}
\delta W_{\text {int }} & =\int_{\Omega_{0} \backslash \Gamma_{0}^{c}}\left(\nabla_{0} \otimes \delta \mathbf{u}\right)^{T}: \mathbf{P} \mathrm{d} \Omega_{0} \\
\delta W_{\text {kin }} & =\int_{\Omega_{0} \backslash \Gamma_{0}^{c}} \varrho_{0} \delta \mathbf{u} \cdot \ddot{\mathbf{u}} \mathrm{d} \Omega_{0} \\
\delta W_{\text {ext }} & =\int_{\Omega_{0} \backslash \Gamma_{0}^{c}} \varrho_{0} \delta \mathbf{u} \cdot \boldsymbol{b} \mathrm{d} \Omega_{0}+\int_{\Gamma_{0}^{t}} \delta \mathbf{u} \cdot \overline{\boldsymbol{t}}_{0} \mathrm{~d} \Gamma_{0} \\
\delta W_{\text {coh }} & =\int_{\Gamma_{0}^{c}} \llbracket \delta \mathbf{u} \rrbracket \cdot \boldsymbol{\tau} \mathrm{d} \Gamma_{0} \\
\delta W_{\mathrm{L}} & =\delta \int_{\Gamma_{0}^{c, \text { ext }}} \boldsymbol{\Lambda} \cdot \llbracket \mathbf{u} \rrbracket \mathrm{d} \Gamma_{0}
\end{aligned}
$$

in which $\delta W_{\mathrm{L}}$ is the general variation with constraint and $\boldsymbol{\Lambda}$ is the Lagrange multiplier vector. As the Lagrange multiplier is defined for $\Gamma_{0}^{c, \text { ext }}$ and discretized using the same shape functions as the enrichment, the discretized Lagrange multiplier is given by

$$
\Lambda=2 \Phi \lambda
$$

where $\boldsymbol{\lambda}$ are the coefficients of the discretization of the Lagrange multiplier $\boldsymbol{\Lambda}$. Substituting the continuous and discontinuous displacement fields $\mathbf{u}^{s}$ and $\mathbf{u}^{e}$ in Eqs. (5) and (7), and the crack opening displacement $\llbracket \mathbf{u} \rrbracket$ in Eq. (13) into the weak form, we obtain ${ }^{7}$

\footnotetext{
$\overline{7}$ we denote by $\Gamma_{0}^{c \alpha}$ the surface of crack $\alpha$ in the reference configuration. The union of the $\Gamma_{0}^{c \alpha}$ for $\alpha \in \mathcal{E}$ forms set $\Gamma_{0}^{c}$.
} 


$$
\begin{aligned}
& \delta W_{\mathrm{int}}=\sum_{I \in \mathcal{W}} \delta \mathbf{u}_{I}^{T} \int_{\Omega_{0} \backslash \Gamma_{0}^{c}} \nabla_{0} \Phi_{I}(\mathbf{X})^{T}: \mathbf{P} \mathrm{d} \Omega \\
& +\sum_{\alpha \in \mathcal{E}} \sum_{K \in \mathcal{W}_{\alpha}} \delta \boldsymbol{a}_{K}^{\alpha T} \int_{\Omega_{0} \backslash \Gamma_{0}^{c \alpha}} \nabla_{0}\left[\Phi_{K}(\mathbf{X}) \Psi_{K}^{\alpha}(\mathbf{X})\right]^{T}: \mathbf{P} \mathrm{d} \Omega \\
& \delta W_{\mathrm{kin}}=\sum_{I \in \mathcal{W}} \delta \mathbf{u}_{I}^{T} \sum_{J \in \mathcal{W}} \int_{\Omega_{0} \backslash \Gamma_{0}^{c}} \varrho_{0} \Phi_{I}(\mathbf{X})^{T} \cdot \Phi_{J}(\mathbf{X}) \mathrm{d} \Omega \ddot{\mathbf{u}}_{J} \\
& +\sum_{I \in \mathcal{W}} \delta \mathbf{u}_{I}^{T} \sum_{\alpha \in \mathcal{E}} \sum_{K \in \mathcal{W}_{\alpha}} \int_{\Omega_{0} \backslash \Gamma_{0}^{c \alpha}} \varrho_{0} \Phi_{I}(\mathbf{X})^{T} \cdot \Phi_{K}(\mathbf{X}) \Psi_{K}^{\alpha}(\mathbf{X}) \mathrm{d} \Omega \ddot{\boldsymbol{a}}^{a} l_{K} \\
& +\sum_{\alpha \in \mathcal{E}} \sum_{K \in \mathcal{W}_{\alpha}} \delta \boldsymbol{a}_{K}^{\alpha T} \sum_{I \in \mathcal{W}} \int_{\Omega_{0} \backslash \Gamma_{0}^{c \alpha}} \varrho_{0}\left[\Phi_{K}(\mathbf{X}) \Psi_{K}^{\alpha}(\mathbf{X})\right]^{T} \cdot \Phi_{I}(\mathbf{X}) \mathrm{d} \Omega \ddot{\mathbf{u}}_{I} \\
& +\sum_{\alpha \in \mathcal{E}} \sum_{K \in \mathcal{W}_{\alpha}} \delta \boldsymbol{a}_{K}^{\alpha T} \sum_{\beta \in \mathcal{E}} \sum_{M \in \mathcal{W}_{\beta}} \\
& \int_{\Omega_{0} \backslash\left(\Gamma_{0}^{c \beta} \cup \Gamma_{0}^{c \alpha}\right)} \varrho_{0}\left[\Phi_{K}(\mathbf{X}) \Psi_{K}^{\alpha}(\mathbf{X})\right]^{T} \cdot \Phi_{M}(\mathbf{X}) \Psi_{M}^{\beta}(\mathbf{X}) \mathrm{d} \Omega \ddot{\boldsymbol{a}}_{M}^{L} \\
& \delta W_{\text {ext }}=\sum_{I \in \mathcal{W}} \delta \mathbf{u}_{I}^{T} \int_{\Omega_{0} \backslash \Gamma_{0}^{c}} \varrho_{0} \Phi_{I}(\mathbf{X})^{T} \cdot \boldsymbol{b} \mathrm{d} \Omega+\sum_{I \in \mathcal{W}} \delta \mathbf{u}_{I}^{T} \int_{\Gamma_{0}^{t}} \Phi_{I}(\mathbf{X})^{T} \cdot \overline{\boldsymbol{t}}_{0} \mathrm{~d} \Gamma(32) \\
& \delta W_{\mathrm{coh}}=2 \sum_{\alpha \in \mathcal{E}} \sum_{K \in \mathcal{W}_{\alpha}} \delta \boldsymbol{a}_{K}^{T} \int_{\Gamma_{0}^{c \alpha}} \Phi_{K}(\mathbf{X})^{T} \cdot \overline{\boldsymbol{t}}_{c} \mathrm{~d} \Gamma \\
& \delta W_{\mathrm{L}}=4 \sum_{\alpha \in \mathcal{E}} \sum_{I \in \mathcal{W}_{\alpha}} \delta \boldsymbol{\lambda}_{I}^{\alpha T} \sum_{\beta \in \mathcal{E}} \sum_{J \in \mathcal{W}_{\beta}} \int_{\Gamma_{0}^{c, \text { ext }}} \Phi_{I}^{\alpha}(\mathbf{X})^{T} \cdot \Phi_{J}^{\beta}(\mathbf{X}) \mathrm{d} \Gamma_{0} \boldsymbol{a}_{J}^{\beta} \\
& +4 \sum_{\alpha \in \mathcal{E}} \sum_{I \in \mathcal{W}_{\alpha}} \boldsymbol{\lambda}_{I}^{\alpha T} \sum_{\beta \in \mathcal{E}} \sum_{J \in \mathcal{W}_{\beta}} \int_{\Gamma_{0}^{c, \text { ext }}} \Phi_{I}^{\alpha}(\mathbf{X})^{T} \cdot \Phi_{J}^{\beta}(\mathbf{X}) \mathrm{d} \Gamma_{0} \delta \boldsymbol{a}_{J}^{\beta}
\end{aligned}
$$

Using the fundamental lemma of calculus, we obtain the discretized equations

$$
\begin{aligned}
\mathbf{M} \ddot{\mathbf{q}} & =\mathrm{f}_{\text {ext }}+\mathrm{f}_{\mathrm{coh}}-\mathrm{f}_{\mathrm{int}}-\mathrm{f}_{\mathrm{L}} \\
\mathbf{G} \mathbf{a} & =\mathbf{0}
\end{aligned}
$$

where $\mathbf{M}$ is the consistent mass matrix, $\mathbf{q}$ is the vector of generalized parameters, $\mathbf{f}_{\text {ext }}, \mathbf{f}_{\text {int }}, \mathbf{f}_{\text {coh }}$ are the discrete external, internal and cohesive force vectors, respectively; $\mathbf{f}_{\mathrm{L}}=\boldsymbol{\lambda}^{T} \mathbf{G}$ in which $\mathbf{G}=4 \int_{\Gamma_{0}^{c, e x t}} \boldsymbol{\Phi}^{T} \boldsymbol{\Phi} \mathrm{d} \Gamma_{0}$ is the force term due to the constraint to close the crack at its front. The expressions for $\mathbf{M}, \mathbf{q}, \mathbf{f}_{\text {ext }}$, $\mathbf{f}_{\text {coh }}$ and $\mathbf{f}_{\text {coh }}$ are given by 


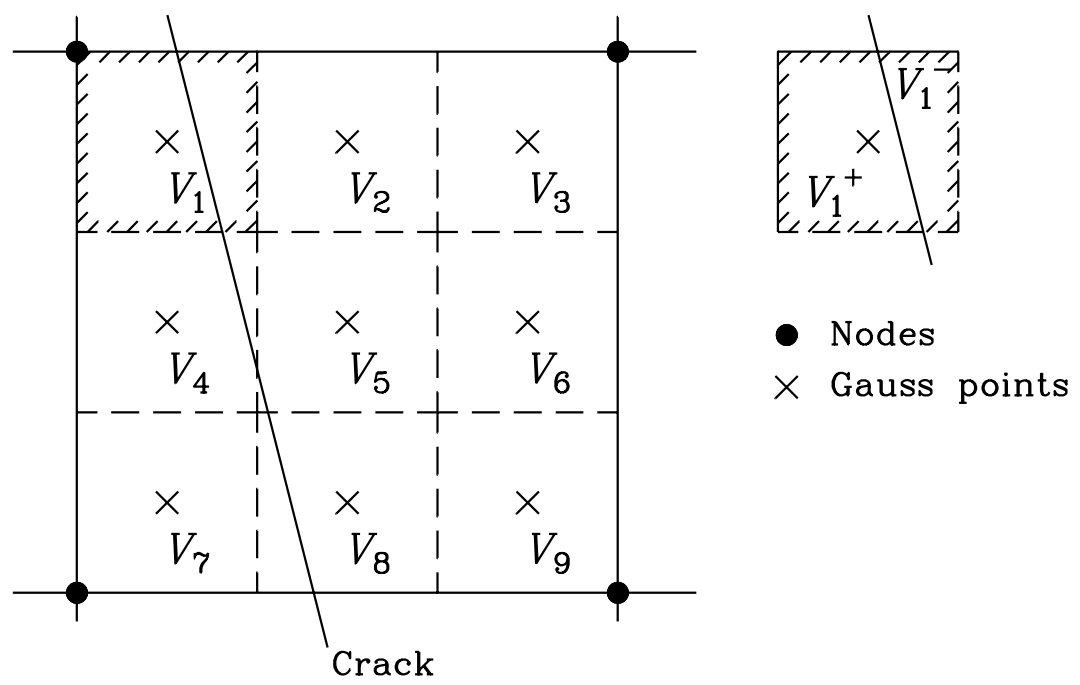

Fig. 9. Numerical Integration

$$
\begin{aligned}
& \mathbf{M}=\int_{\Omega_{0} \backslash \Gamma_{0}^{c}} \varrho_{0}\left[\begin{array}{cc}
\boldsymbol{\Phi}^{0^{T}} \boldsymbol{\Phi}^{0} & \boldsymbol{\Phi}^{0^{T}} \boldsymbol{\Phi}^{e} \\
\boldsymbol{\Phi}^{e T} \boldsymbol{\Phi}^{0} & \boldsymbol{\Phi}^{e T} \boldsymbol{\Phi}^{e}
\end{array}\right] \mathrm{d} \Omega \\
& \mathbf{f}_{\mathrm{int}}=\int_{\Omega_{0} \backslash \Gamma_{0}^{c}} \mathbf{B}^{0^{T}} \mathbf{P} \mathrm{d} \Omega+\int_{\Omega_{0} \backslash \Gamma_{0}^{c}} \mathbf{B}^{e T} \mathbf{P} \mathrm{d} \Omega \\
& \mathbf{f}_{\text {ext }}=\int_{\Omega_{0} \backslash \Gamma_{0}^{c}} \varrho_{0} \boldsymbol{\Phi}^{T} \mathbf{b} \mathrm{d} \Omega+\int_{\Gamma_{0}^{t}} \boldsymbol{\Phi}^{T} \overline{\mathbf{t}}_{0} \mathrm{~d} \Gamma \\
& \mathbf{f}_{\mathrm{coh}}=2 \int_{\Gamma_{0}^{c}} \boldsymbol{\Phi}^{T} \mathbf{t}_{c 0} \mathrm{~d} \Gamma \\
& q=\left\{\begin{array}{l}
u \\
a
\end{array}\right\} \\
& \mathbf{u}=\left[\mathbf{u}_{I}^{s}\right]^{T} \quad \forall I \in \mathcal{W} \text { and } \mathbf{a}=\left[\boldsymbol{a}_{K}^{\alpha}\right]^{T} \quad \forall K \in \mathcal{W}_{\alpha}, \forall \alpha \in \mathcal{E} \\
& \boldsymbol{\Phi}^{0}=\left[\boldsymbol{\Phi}_{I}\right] \quad \forall I \in \mathcal{W} \text { and } \boldsymbol{\Phi}^{e}=\left[\boldsymbol{\Phi}_{\alpha} \boldsymbol{\Psi}_{K}^{\alpha}\right] \quad \forall K \in \mathcal{W}_{\alpha}, \forall \alpha \in \mathcal{E} \\
& \mathbf{B}^{0}=\nabla_{0} \boldsymbol{\Phi}^{0} \text { and } \mathbf{B}^{e}=\nabla_{0} \boldsymbol{\Phi}^{e}
\end{aligned}
$$

\subsection{Numerical integration}

To integrate the discrete equations (37) to (40), tetrahedral background integration cells are constructed. If no crack crosses an integration cell, standard Gauss quadrature is sufficient. Instead of using a sub-tetrahedration procedure, we use a procedure described in Rabczuk and Areias [27] and modify 

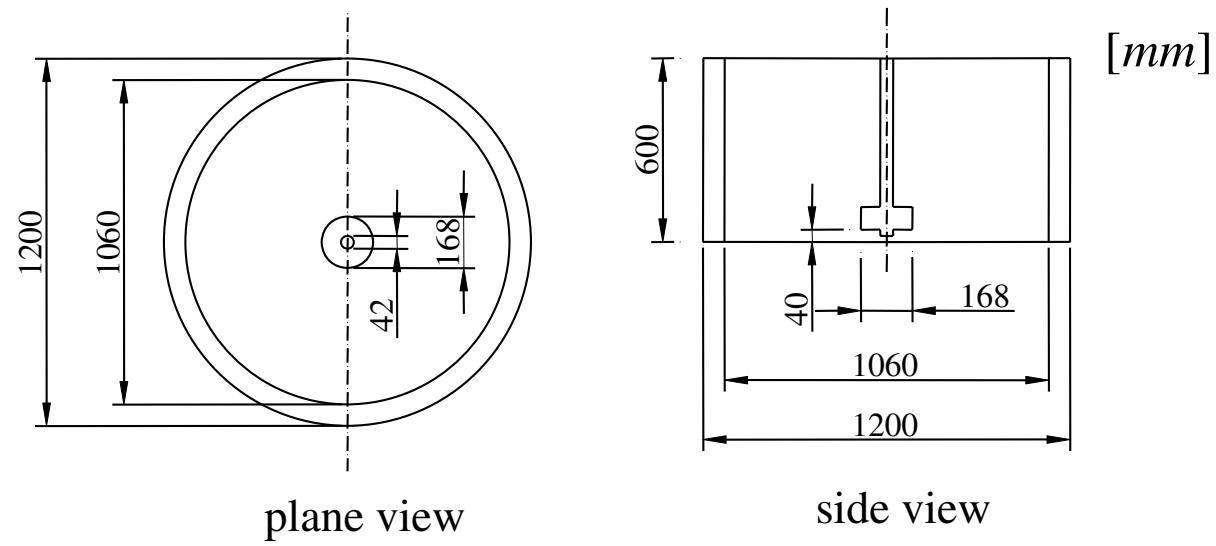

Fig. 10. Test-setup of the Pull out test

the quadrature weights according to Figure 9. Since in meshfree methods, a larger number of Gauss points is needed for accurate integration, this procedure is preferable since the mapping of quantities and state variables that occurs when a crack enters a background cell and that generates additional inaccuracies ${ }^{8}$ can be omitted. The modified quadrature weights are easily obtained by a "local" Voronoï procedure ${ }^{9}$ on "background element level" followed by the determination of the crack intersections and the volumes $V^{+}$and $V^{-}$as shown in Figurebubel.

Note that since there is no near-tip enrichment, no special care is required to integrate the non-polynomial, or high-order polynomial fields that were used in [26], for instance.

\section{Numerical Examples}

\subsection{Pull-out test}

Consider a pull out test of reinforced concrete as shown in Figure 10. This example was studied previously by Gasser and Holzapfel [39] and Areias and Belytschko [40] by the PUFEM and XFEM, respectively. We also employed symmetry conditions and modeled only one quarter of the specimen. A vertical displacement boundary condition is applied to pull the reinforcement bar out of the concrete specimen as illustrated in Figure 10. We adopted the same constitutive and cohesive model as [39]. At crack initiation, the cohesive traction are computed from the bulk, i.e. $\mathbf{t}_{c}=\mathbf{n} \cdot \boldsymbol{\sigma}$ to ensure traction continuity. The material parameters are $\kappa=16,670 \mathrm{MPa}$ and $\nu=12,500 \mathrm{MPa}$. For

\footnotetext{
8 due to the mapping

9 that is already implemented in most meshfree codes.
} 


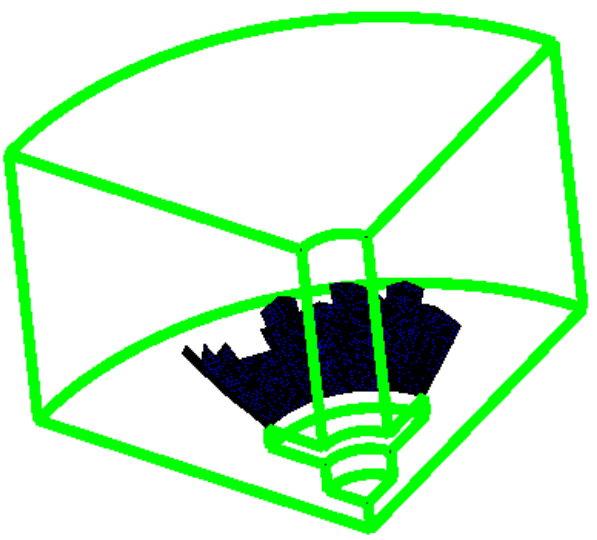

(a)

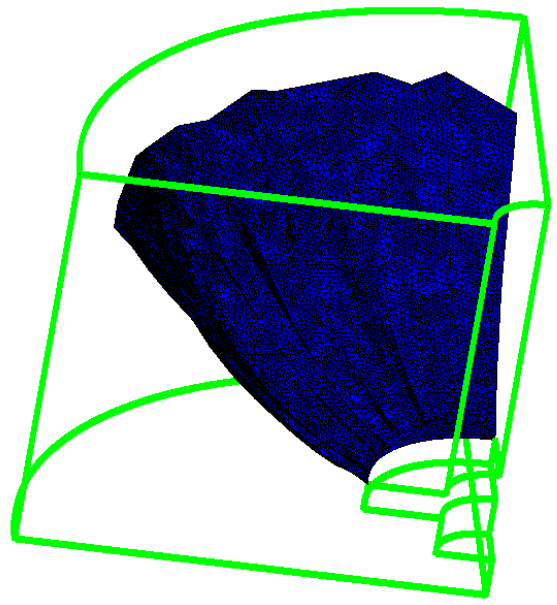

(c)

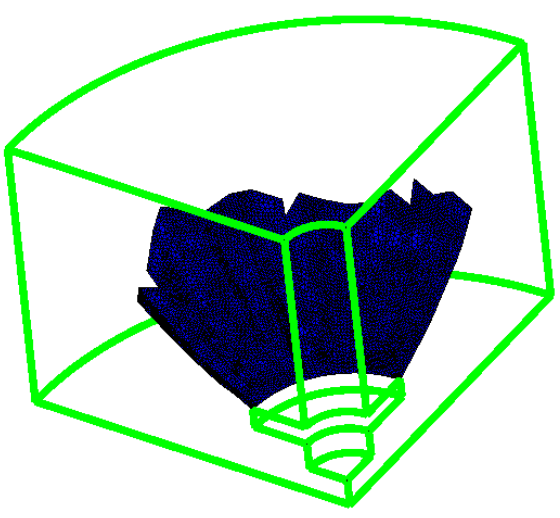

(b)

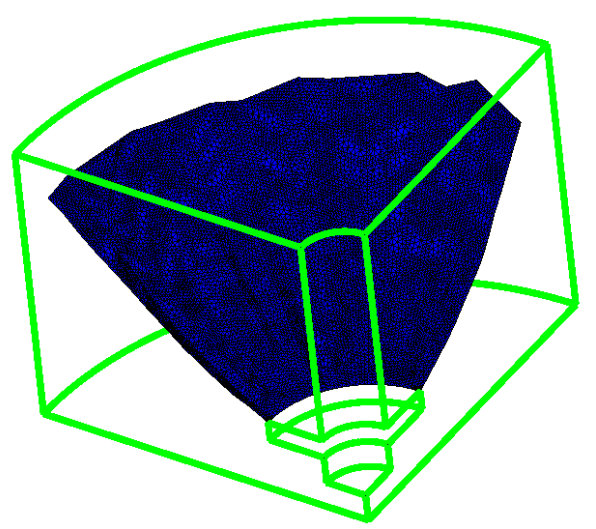

(d)

Fig. 11. Crack pattern of the pull-out test

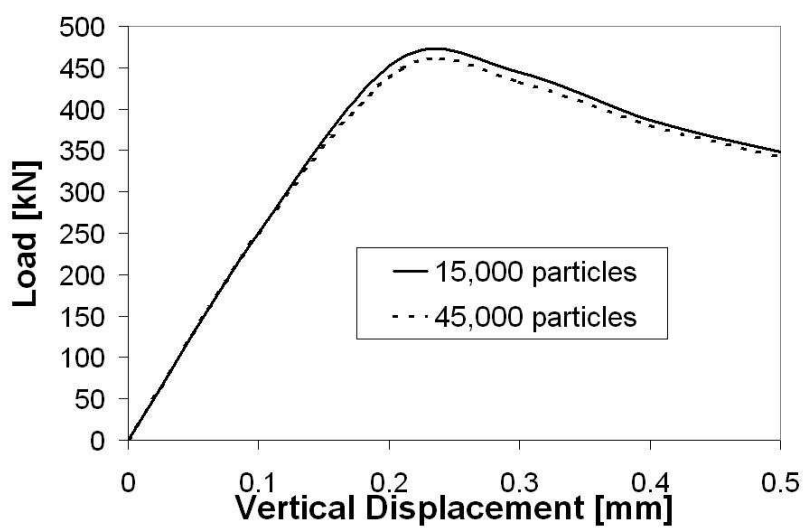

Fig. 12. Load deflection curves of the pull-out test for different refinement 

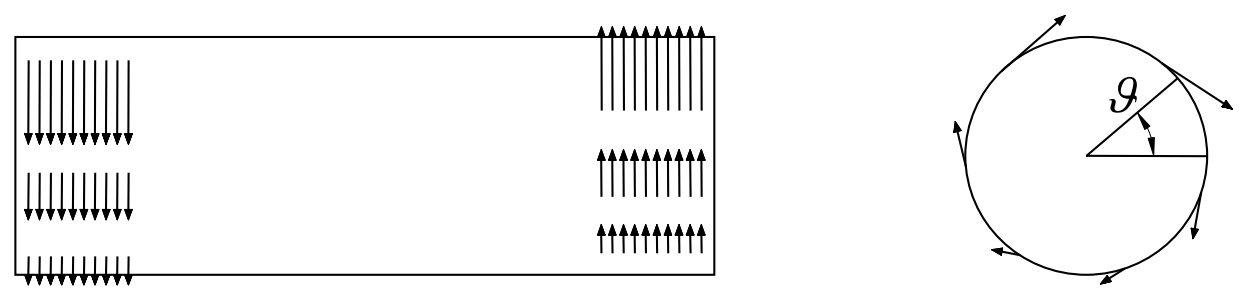

Fig. 13. Set-up of the chalk torsion test.

the cohesive model, we use, according to [39], $t_{0}=3 \mathrm{MPa}, a=11.323 \mathrm{~mm}^{-1}$, $b=0.674$ and $\alpha=1$.

We tested two discretizations starting with 15,000 and 45,000 nodes and refined adaptively [41]. The crack pattern at different load stages is shown in Figure 11 for the fine discretization and different view points. Note that the surface of the crack is rippling, and is captured nicely. The load-deflection curves for two different refinement are shown in Figure 12 and is similar to the results presented in $[26,39,40]$.

\subsection{Chalk under torsion}

In this section, we analyze numerically the problem of a circular-cylindrical chalk bar under torsion. The test setup is shown in Figure 13. A non-uniform traction boundary condition is applied and a small pertubation is introduced on the lateral surface on the midplane normal to the cylinder's director. The traction is applied in the tangential direction to the surface of the chalk and its magnitude $t$ is given by

$$
t=t_{\max } \sin \left(\frac{\vartheta+\frac{\pi}{2}}{2}\right)
$$

in which $t_{\max }$ is the maximum traction and $\vartheta$ is the angle as shown in Figure 13. The material properties used are $E=2 \mathrm{GPa}, \nu=0.18$ and $G_{f}=50 \mathrm{~N} / \mathrm{m}$. The linear cohesive law is adopted for simplicity. $t_{\max }$ was $200 \mathrm{kPa}$.

If the numerical chalk specimen were "perfect", the failure would happen at an arbitrary position in the longitudinal direction of the chalk. The experimental failure surface of the chalk is compared to the numerical failure surface in Figure 14 . We note that the numerical results are relatively sensitive to boundary conditions and to the dimensions of the model specimen; more specifically, the ratio between the length and the cross section is influencial. If the length is large compared to the diameter of the chalk bar, and the tractions applied uniformly, then the elastic strain energy stored is large, resulting in a relatively straight fracture surface. For non-uniform boundary conditions, as illustrated in Figure 14, we obtain a hellical fracture surface, comparable to 


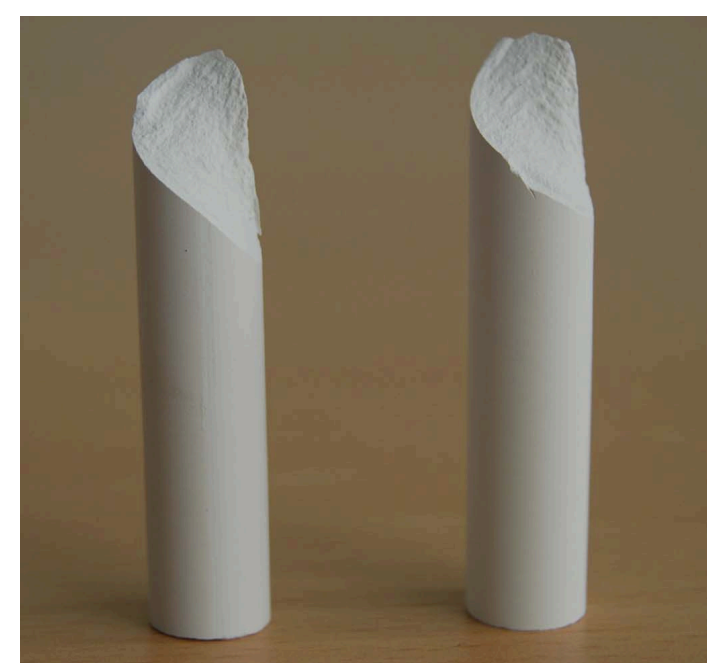

(a)

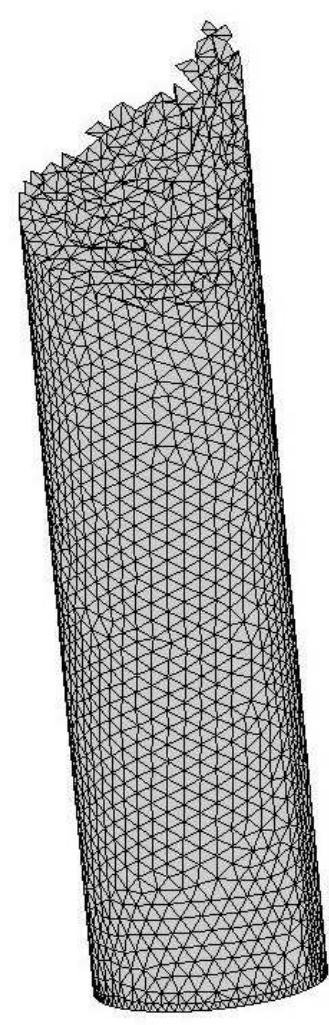

(b)

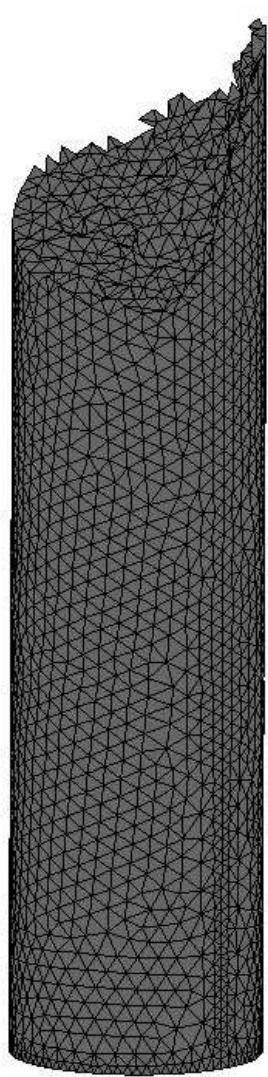

(c)

Fig. 14. Chalk bar under torsion in which the simulation result was depicted using the integration cells. 
$\sigma$

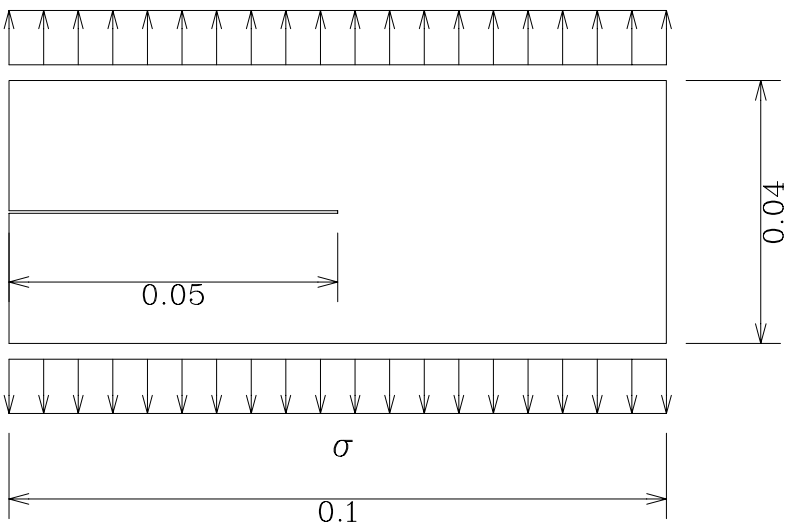

Fig. 15. A plate with an edge crack loaded by a uniform traction on the top and bottom edges.

the experimental failure surface.

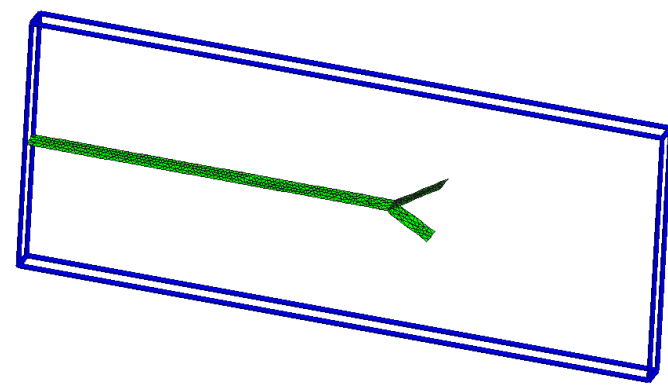

(a) $t=30 \mathrm{~ms}$

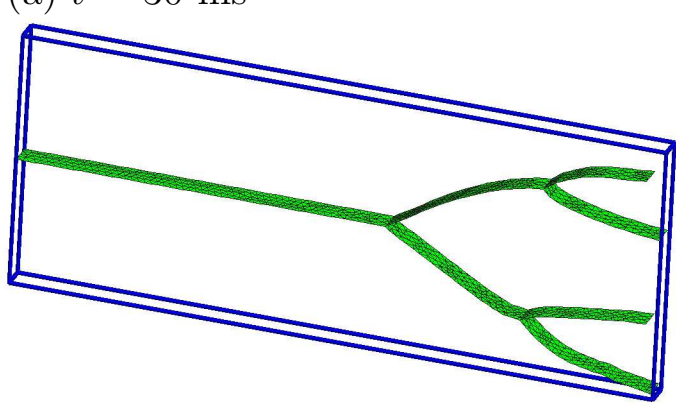

(c) $t=60 \mathrm{~ms}$

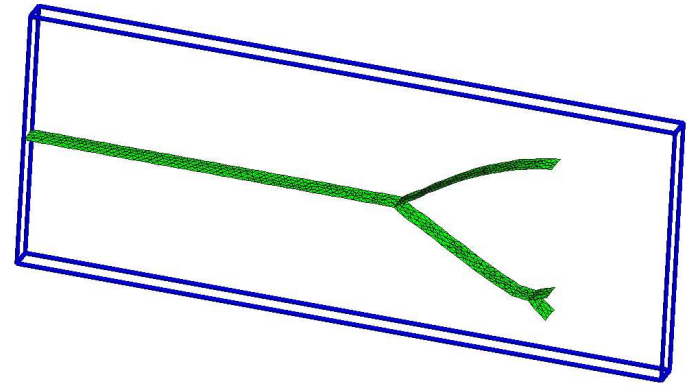

(b) $t=45 \mathrm{~ms}$

Fig. 16. Cracking branching at different time steps.

\subsection{Crack branching}

In this section, we examine the performance of the method for a crack branching problem. Consider a rectangular prenotched specimen as shown in Figure 15. The length of the rectangle is $0.1 \mathrm{~m}$, the width $0.04 \mathrm{~m}$ and depth 0.004 $\mathrm{m}$. Initially, there is a horizontal crack from the left edge to the center of the 


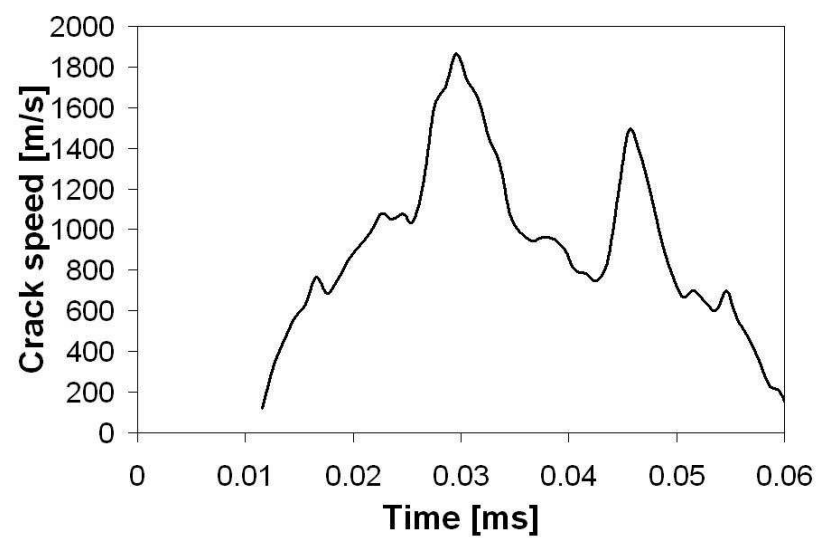

Fig. 17. Crack speed time history for the crack branching problem in which the crack branches as the speed reached the peaks. 
plate over the entire thickness. A tensile traction of $1 \mathrm{MPa}$ is applied on the top and bottom edges.

We used Lemaitre's damage law [42], loss of hyperbolicity and an exponential decaying cohesive law (Figure 8c). The material constants are $\varrho=2,450$ $\mathrm{kg} / \mathrm{m}^{3}, E=32 \mathrm{MPa}, \nu=0.2$ and $A=1.0, B=7,300$ and $\epsilon_{D_{0}}=8.5 \times$ $10^{-5}$ for the Lemaitre model. Two dimensional computations of this problem was previously reported in $[43,44,45]$. A three dimensional computation was carried out in [46]. Experimental data of this problem is available; see [47, 48, 49].

The crack pattern is shown in Figure 16 at different time steps. Two crack branches appear, similar to the previous results in $[45,46]$. The time history of the crack speed is shown in Figure 17. The crack starts to propagate at about $0.012 \mathrm{~ms}$ and the crack speed increases almost up to the maximum theoretical value of the Rayleigh wave speed. Then the crack branches and the crack speed quickly decreases again. A similar result was reported by Belytschko et al. [50]. Following the upper path of the $\mathrm{crack}^{10}$, the crack speed soon accelerates and at a certain speed, the crack branches again. Directly after crack branching, the crack speed decreases monotonically until the crack almost hits the right hand end of the specimen. We note that the crack speed at the second time of bifurcation is smaller compared to the one when the crack bifurcates first. The relation between crack speed and crack branching is still an unsolved problem.

\subsection{Taylor bar impact}

To test the method for multiple cracks with crack junction, we consider a Taylor bar impact. There are experimental results available; see Teng et al. [51]. The Taylor bar has a diameter of $6 \mathrm{~mm}$ and length $30 \mathrm{~mm}$. We consider an impact velocities of $600 \mathrm{~m} / \mathrm{s}$. The failure mechanism is petalling. We have performed similar computations in [26] but for a much smaller impact velocity where relatively moderate strains occur. The material is a 2024-T351 aluminium alloy. We use the Johnson Cook model [52] as in the previous section with Young's modulus $E=74 \mathrm{GPa}$, Poisson ratio $\nu=0.3$, density 2,700 $\mathrm{kg} / \mathrm{m}^{3}$, a reference strain rate of $3.33 \times 10^{-4}, A=352 \mathrm{MPa}, B=440 \mathrm{MPa}$, $C=0.0083, n=0.42, m=1, c_{v}=875 \mathrm{~J} / \mathrm{kg}{ }^{\circ} \mathrm{C}, T_{r}=296 \mathrm{~K}, T_{m}=775 \mathrm{~K}$ and $\beta=1$. We tested two different discretizations, with approximately 7,000 particles and 22,000 particles. The final deformation of the Taylor bar is shown in Figure 18 for both discretizations. As can be seen, multiple cracking occurs including crack junctions. A similar failure mode was observed in Teng et al. [51]. There are basically four major cracks that cause the petalling. Our failure mode is slightly too ductile, which is likely related to difficulties in the

\footnotetext{
$\overline{10}$ the same observations apply for the lower crack path
} 


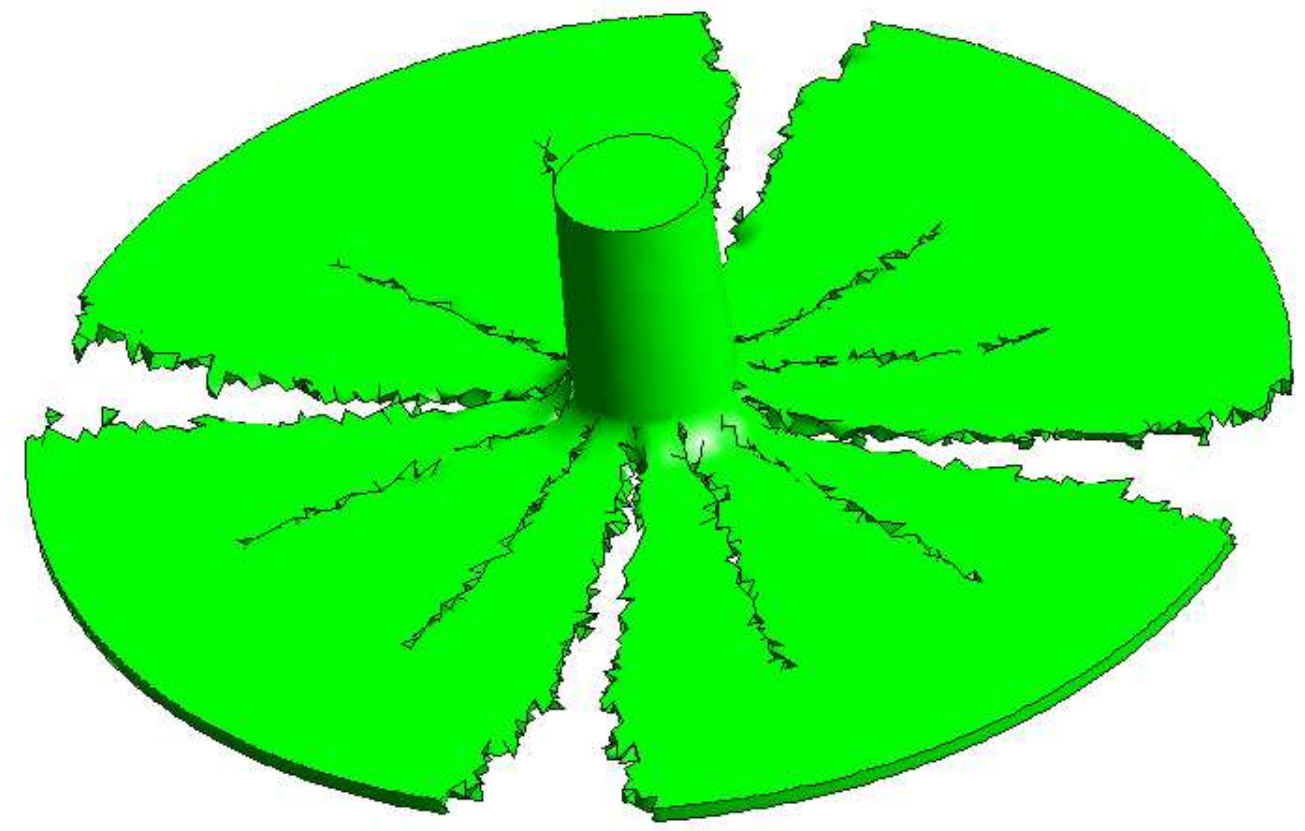

a) 22,000 particles

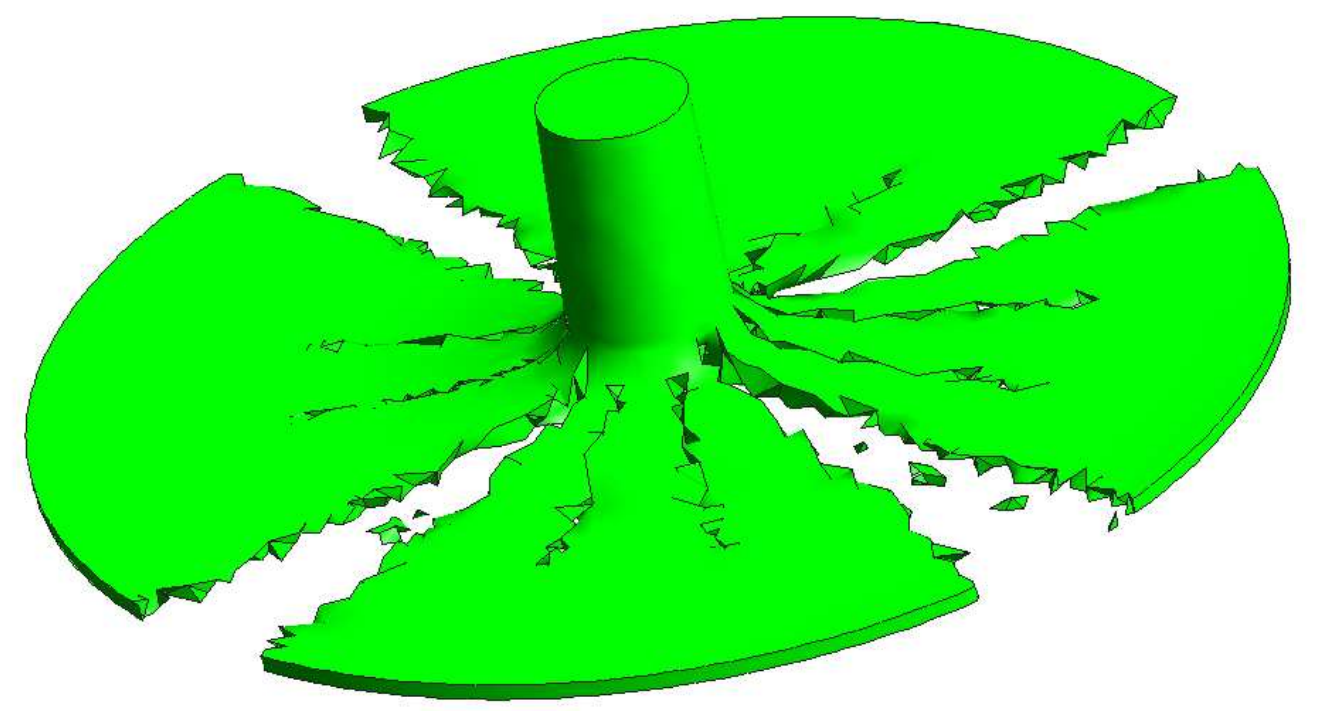

b) 7,000 particles

Fig. 18. Final crack pattern of the Taylor bar problem 
discontinuous bifurcation analysis since the Johnson Cook material does not lose stability easily. The results look almost identical for both discretizations, and agree well with the experimental data in [51].

\section{Conclusions}

This paper presented a three-dimensional adaptive meshfree method for fracture in statics and dynamics. The initiation, growth, coallescence and branching of an arbitrary number of cracks is handled simply and effectively.

The discontinuities are introduced through extrinsic enrichment of the moving least squares basis, but no near-front enrichment is required. To close the cracks, a Lagrange multiplier field is instead added along the front of the cracks. Geometrically, the cracks are non-planar surfaces composed of triangular and quadrangular planar sections obtained by cutting the tetrahedral background integration cells by planes whose normals are provided by a material stability analysis.

The results show accurate simulations of large deformation failure problems including fragmentation, where the flexibility of the meshfree method coupled with the efficient crack interaction procedure is most clear. The simulations agree well with experimental results available in the literature.

\section{Nomenclature}

$H \quad$ The Heaviside function

I The index of a particle

$W \quad$ The weight or kernel function

$\Gamma_{c}^{\alpha} \quad$ The surface of crack $\alpha$

$\Gamma_{0}^{t} \quad$ The traction boundary

$\Gamma_{0}^{u} \quad$ The displacement boundary

$\Omega_{0} \quad$ The body of interest in the reference configuration

$\mathbf{P}$ The nominal stress tensor

$\Phi_{I} \quad$ The MLS shape function associated with particle $I$

$\Psi_{I}^{\alpha} \quad$ The enrichment function associated with particle $I$ and crack $\alpha$

$\mathbf{X}$ A point in the reference configuration

$\mathbf{X}_{\alpha} \quad$ A point on the surface $\Gamma_{c}^{\alpha}$ of crack $\alpha$

$\alpha \quad$ The index of a crack

$\bar{r}_{I} \quad$ The dimensionless distance

$\overline{\boldsymbol{t}} \quad$ The prescribed traction on the traction boundary

$\overline{\mathbf{u}} \quad$ The prescribed displacement on the displacement boundary

$\mathbb{R} \quad$ The Euclidian space 
$\boldsymbol{\Lambda} \quad$ The Lagrange multiplier

$\boldsymbol{Q}$ The acoustic tensor

$\boldsymbol{\sigma}$ The Cauchy stress tensor

$\tau$ The cohesive traction

$\mathcal{E} \quad$ The set of all the cracks in the domain

$\mathcal{I}_{I \mathbf{X}}$ the set of $N_{I \mathbf{X}}$ particles whose domain of influence contain a point $\mathbf{X}$

$\mathcal{W}$ The set of all particles in $\Omega_{0}$

$\ddot{\mathbf{u}} \quad$ The acceleration

$\delta W_{\text {int }}, \delta W_{\text {kin }}, \delta W_{\text {ext }}, \delta W_{\text {coh }}, W_{\mathrm{L}}$ The virtual work of the internal stress, the inertia force, the external traction, the cohesive traction and the Lagrange multiplier vector, respectively

$\delta_{n} \quad$ The mode $I$ crack opening displacement

$\delta_{t} \quad$ The mode $I I$ crack opening displacement

$\delta \mathbf{u}$ The variation of displacement vector

$\mathcal{W}_{b}^{\alpha} \quad$ The set of nodes whose domain of influence is cut by crack $\alpha$

n The crack normal

p The basis vector for the moving least square approximation

u The displacement field

$\mathbf{u}^{e} \quad$ The enriched (or discontinuous) displacement field

$\mathbf{u}^{s} \quad$ The standard (or continuous) displacement field

$\varrho_{0} \quad$ The initial mass density

$f^{\alpha}(\mathbf{X})$ The signed distance from $\mathbf{X}$ to crack $\alpha$

$h_{I} \quad$ The approximation radius of particle $I$

$r_{I} \quad$ The distance between $\mathbf{X}$ and particle $I$

$t \quad$ The time

M The consistent mass matrix

a The discrete vector of the additional parameters for the enrichment

$\mathbf{f}_{\mathrm{L}} \quad$ The discrete force vector for the Lagrange multiplier

$\mathbf{f}_{\text {ext }}, \mathbf{f}_{\mathrm{int}}, \mathbf{f}_{\mathrm{coh}}$ The discrete external, internal and cohesive force vectors, respectively

q The vector of generalized parameters

$\boldsymbol{C}^{t} \quad$ The fourth order tangential modulus

$\boldsymbol{\lambda}$ The coefficients of the discretization of the Lagrange multiplier $\boldsymbol{\Lambda}$

$\boldsymbol{a}_{I}^{\alpha} \quad$ The additional degrees of freedom for the enrichment $\Psi_{I}^{\alpha}$ 


\section{References}

[1] T. Belytschko, T. Black, Elastic crack growth in finite elements with minimal remeshing, International Journal for Numerical Methods in Engineering 45 (5) (1999) 601-620.

[2] N. Moës, J. Dolbow, T. Belytschko, A finite element method for crack growth without remeshing, International Journal for Numerical Methods in Engineering 46 (1) (1999) 133-150.

[3] N. Moës, A. Gravouil, T. Belytschko, Non-planar 3-D crack growth by the extended finite element method and level sets, part I: Mechanical model, International Journal for Numerical Methods in Engineering 53 (11) (2002) 2549-2568.

[4] G. Zi, T. Belytschko, New crack-tip elements for xfem and applications to cohesive cracks, International Journal for Numerical Methods in Engineering 57 (15) (2003) 2221-2240.

[5] G. Zi, J.-H. Song, E. Budyn, S.-H. Lee, T. Belytschko, A method for growing multiple cracks without remeshing and its application to fatigue crack growth, Modelling and Simulation in Materials Science and Engineering 12 (1) (2004) 901-915.

[6] P. M. A. Areias, , T. Belytschko, Non-linear analysis of shells with arbitrary evolving cracks using XFEM, International Journal for Numerical Methods in Engineering 62 (2005) 384-415.

[7] A. Gravouil, N. Moës, T. Belytschko, Non-planar 3D crack growth by the extended finite element and level sets - part ii: Level set update, International Journal for Numerical Methods in Engineering 53 (2002) 2569-2586.

[8] S. Bordas, M. Duflot, Derivative recovery and a posteriori error estimation in extended finite element methods, Computer Methods in Applied Mechanics and Engineering (2007) in press.

[9] M. Duflot, S. Bordas, An extended global recovery procedure for a posteriori error estimation in extended finite element methods, International Journal for Numerical Methods in Engineering (2007) in press.

[10] T. Belytschko, H. Chen, J. Xu, G. Zi, Dynamic crack propagation based on loss of hyperbolicity and a new discontinuous enrichment, International Journal for Numerical Methods in Engineering 58 (12) (2003) 18731905.

[11] G. Zi, H. Chen, J. Xu, T. Belytschko, The extended finite element method for dynamic fractures, Shock and Vibration 12 (1) (2005) 9-23.

[12] J.-H. Song, P. M. A. Areias, T. Belytschko, A method for dynamic crack and shear band propagation with phantom nodes, International Journal for Numerical Methods in Engineering 67 (2006) 868-893.

[13] J. Réthoré, A. Gravouil, A. Combescure, An energy-conserving scheme for dynamic crack growth using the eXtended fnite element method, International Journal for Numerical Methods in Engineering 63 (2005) 631-659.

[14] S. Bordas, B. Moran, Enriched finite elements and level sets for damage 
tolerance assessment of complex structures, Engineering Fracture Mechanics 73 (2006) 1176-1201.

[15] S. Bordas, J. Conley, B. Moran, J. Gray, E. Nichols, A simulation-based design paradigm for complex cast components, Engineering with ComputersIn press, online first.

[16] S. Bordas, M. Duflot, P. Le, A simple a posteriori error estimator for the extended finite element method, Computer Methods in Applied Mechanics and Engineering (2007) in press.

[17] S. Bordas, V. Nguyen, C. Dunant, A. Guidoum, H. Nguyen-Dang, An extended finite element library, International Journal for Numerical Methods in EngineeringIn press, available online.

[18] C. Dunant, S. Bordas, P. Nguyen, A. Guidoum, H. Nguyen-Dang, Architecture trade-offs of including a mesher in an object-oriented extended finite element code., European Journal of Computational MechanicsIn press.

[19] T. Belytschko, M. Tabbara, Dynamic fracture using element-free galerkin methods, International Journal for Numerical Methods in Engineering 39 (6) (1996) 923-938.

[20] Y. Lu, T. Belytschko, M. Tabbara, Element-free galerkin method for wave-propagation and dynamic fracture, Computer Methods in Applied Mechanics and Engineering 126 (1-2) (1995) 131-153.

[21] T. Belytschko, Y. Lu, Element-free galerkin methods for static and dynamic fracture, International Journal of Solids and Structures 32 (1995) 2547-2570.

[22] T. Belytschko, Y. Lu, L. Gu, Crack propagation by element-free galerkin methods, Engineering Fracture Mechanics 51 (2) (1995) 295-315.

[23] P. Krysl, T. Belytschko, The element free galerkin method for dynamic propagation of arbitrary 3-D cracks, International Journal for Numerical Methods in Engineering 44 (6) (1999) 767-800.

[24] G. Ventura, J. Xu, T. Belytschko, A vector level set method and new discontinuity approximations for crack growth by efg, International Journal for Numerical Methods in Engineering 54 (6) (2002) 923-944.

[25] T. Rabczuk, G. Zi, A meshfree method based on the local partition of unity for cohesive cracks, Computational Mechanics 39 (6) (2007) 743760.

[26] T. Rabczuk, S. Bordas, G. Zi, A three-dimensional meshfree method for continuous multiple-crack initiation, propagation and junction in statics and dynamics, Computational Mechanics (2006) -in online.

[27] T. Rabczuk, P. Areias, A meshfree thin shell for arbitrary evolving cracks based on an external enrichment, Computer Modeling in Engineering and Sciences.

[28] T. Belytschko, Y. Lu, L. Gu, Element-free galerkin methods, International Journal for Numerical Methods in Engineering 37 (1994) 229-256.

[29] M. Fleming, Y. A. Chu, B. Moran, T. Belytschko, Enriched element-free Galerkin methods for crack tip fields, International Journal for Numerical 
Methods in Engineering 40 (1997) 1483-1504.

[30] T. Strouboulis, K. Copps, I. Babuška, The generalized finite element method: An example of its implementation and illustration of its performance, International Journal for Numerical Methods in Engineering 47 (8) (2000) 1401-1417.

[31] J. Chessa, H. Wang, T. Belytschko, On the construction of blending elements for local partition of unity enriched finite elements., International Journal for Numerical Methods in Engineering 57 (7) (2003) 1015-1038.

[32] F. Stazi, E. Budyn, J. Chessa, T. Belytschko, XFEM for fracture mechanics with quadratic elements, Computational Mechanics 31 (2003) 38-48.

[33] J. Dolbow, A. Devan, Enrichment of enhanced assumed strain approximation for representing strong discontinuities: Addressing volumetric incompressibility and the discontinuous path test, International Journal for Numerical Methods in Engineering 59 (2003) 47-67.

[34] G. Zi, T. Rabczuk, W. Wall, Extended meshfree methods without the branch enrichment for cohesive cracks, Computational Mechanics (2007) -in online.

[35] G. Zi, T. Belytschko, New crack-tip elements for XFEM and applications to cohesive cracks, International Journal for Numerical Methods in Engineering 57 (2003) 2221-2240.

[36] M. Ortiz, A. Pandolfi, Finite-deformation irreversible cohesive elements for three-dimensional crack-propagation analysis, International Journal for Numerical Methods in Engineering 44 (1999) 1267-1282.

[37] Z. P. Bažant, F. C. Caner, Microplane model M5 with kinematic and static constraints for concrete fracture and anelasticity. I: Theory, ASCE Journal of Engineering Mechanics 131 (1) (2005) 31-40.

[38] G. T. Camacho, M. Ortiz, Computational modeling of impact damage in brittle materials., International Journal of Solids and Structures 33 (1996) 2899-2938.

[39] T. Gasser, G. Holzapfel, Modeling 3D crack propagation in unreinforced concrete using pufem, Computer Methods in Applied Mechanics and Engineering 194 (2005) 2859-2896.

[40] P. Areias, T. Belytschko, Analysis of three-dimensional crack initiation and propagation using the extended finite element method, International Journal for Numerical Methods in Engineering 63 (2005) 760-788.

[41] T. Rabczuk, T. Belytschko, Adaptivity for structured meshfree particle methods in 2D and 3D, International Journal for Numerical Methods in Engineering 63 (11) (2005) 1559-1582.

[42] J. Lemaitre, Evaluation of dissipation and damage in metal submitted to dynamic loading, Proceedings ICM 1.

[43] X.-P. Xu, A. Needleman, Numerical simulations of fast crack growth in brittle solids, Journal of the Mechanics and Physics of Solids 42 (1994) 1397-1434.

[44] M. Falk, A. Needleman, J. Rice, A critical evaluation of cohesive zone models of dynamic fracture, Journal of Physics IV 11 (PR5) (2001) 43- 
50 .

[45] T. Rabczuk, T. Belytschko, Cracking particles: A simplified meshfree method for arbitrary evolving cracks, International Journal for Numerical Methods in Engineering 61 (13) (2004) 2316-2343.

[46] T. Rabczuk, T. Belytschko, A three dimensional large deformation meshfree method for arbitrary evolving cracks, Computer Methods in Applied Mechanics and Engineering.

[47] K. Ravi-Chandar, Dynamic fracture of nominally brittle materials, International Journal of Fracture 90 (1-2) (1998) 83-102.

[48] E. Sharon, J. Fineberg, Microbranching instability and the dynamic fracture of brittle materials, Physical Review B 54 (10) (1996) 7128-7139.

[49] J. Fineberg, E. Sharon, G. Cohen, Crack front waves in dynamic fracture, International Journal of Fracture 121 (1-2) (2003) 55-69.

[50] T. Belytschko, H. Chen, J. Xu, G. Zi, Dynamic crack propagation based on loss of hyperbolicity with a new discontinuous enrichment, International Journal for Numerical Methods in Engineering 58 (12) (2003) 18731905.

[51] X. Teng, T. Wierzbicki, S. Hiermaier, I. Rohr, Numerical prediction of fracture in the taylor test, International Journal of Solids and Structures 42 (2005) 2929-2948.

[52] G. Johnson, W. Cook, A constitutive model and data for metals subjected to large strains, high strain rates, and high temperatures, in: Proc. 7th International Symp. on Ballistics, 1983. 